TRANSACTIONS OF THE

AMERICAN MATHEMATICAL SOCIETY

Volume 356, Number 4, Pages 1463-1487

S 0002-9947(03)03459-7

Article electronically published on November 4, 2003

\title{
LS-CATEGORY OF COMPACT HAUSDORFF FOLIATIONS
}

\author{
HELLEN COLMAN AND STEVEN HURDER
}

\begin{abstract}
The transverse (saturated) Lusternik-Schnirelmann category of foliations, introduced by the first author, is an invariant of foliated homotopy type with values in $\{1,2, \ldots, \infty\}$. A foliation with all leaves compact and Hausdorff leaf space $M / \mathcal{F}$ is called compact Hausdorff. The transverse saturated category cat $_{巾} M$ of a compact Hausdorff foliation is always finite.

In this paper we study the transverse category of compact Hausdorff foliations. Our main result provides upper and lower bounds on the transverse category $\operatorname{cat}_{\pitchfork}(M)$ in terms of the geometry of $\mathcal{F}$ and the Epstein filtration of the exceptional set $\mathcal{E}$. The exceptional set is the closed saturated foliated space which is the union of the leaves with non-trivial holonomy. We prove that

$$
\max \left\{\operatorname{cat}(M / \mathcal{F}), \operatorname{cat}_{巾}(\mathcal{E})\right\} \leq \operatorname{cat}_{巾}(M) \leq \operatorname{cat}_{巾}(\mathcal{E})+q .
$$

We give examples to show that both the upper and lower bounds are realized, so the estimate is sharp. We also construct a family of examples for which the transverse category for a compact Hausdorff foliation can be arbitrarily large, though the category of the leaf spaces is constant.
\end{abstract}

\section{INTRODUCTION}

The Lusternik-Schnirelmann category of a topological space $X$ is the least integer $k$ such that $X$ may be covered by $k$ open subsets which are contractible in $X$. This concept was introduced in the course of research on the calculus of variations in 1930 [28, 23, 24]. Extensions of the LS category have been given for actions of compact groups [14, 15] 29], and for fibrewise spaces [25].

The transverse Lusternik-Schnirelmann category of a foliated manifold $(M, \mathcal{F})$ was introduced by Colman in [5, 9, 6]. The key concept is that an open set $U \subset M$ is transversely categorical if the inclusion factors through a leaf, up to foliated homotopy. A foliation chart for $\mathcal{F}$ is always transversely categorical, so for $M$ compact the transverse category of $\mathcal{F}$ is always finite.

A subset of $M$ is saturated if it is a union of leaves of $\mathcal{F}$. The open sets in a transversely categorical covering need not be saturated. A homotopy of an open saturated set to a single leaf is a much stronger condition, as there are geometric and topological constraints on the homotopy. In particular, the saturation of a transversely categorical open set need not be transversely categorical.

The transverse saturated Lusternik-Schnirelmann category cat ${ }_{\pitchfork}(M)$ of $(M, \mathcal{F})$ is the least integer $k$ such that $M$ may be covered by $k$ open saturated subsets which

Received by the editors August 1, 2002.

2000 Mathematics Subject Classification. Primary 55M30, 57R30; Secondary 57S05, 57N80.

The first author was partially supported by grants from the Xunta Galicia, Spain, and University of Sheffield, EU RTN1-1999-00176, Modern Homotopy Theory.

The second author was partially supported by NSF Grant DMS-9704768. 
are transversely categorical in $M$. If no such covering exists, then the transverse saturated category is defined to be infinity.

Clearly, the transverse saturated category is greater than or equal to the transverse category. Both definitions of transverse category are invariants of foliated homotopy.

For a foliation defined by a fibration, its transverse saturated category is just the category of the leaf (or base) space $M / \mathcal{F}$. (Surprisingly, this property has not been proven for the transverse category.) For a foliation defined by a smooth action of a compact connected Lie group $G$, its transverse saturated category may be compared with the $G$-equivariant category introduced by Fadell [14, 29]. For a finite group $G$, the ideas of this paper have been used by the first author in [7] to obtain new estimates of the $G$-category, analogous to our main estimate in Theorem6.1.

A foliation $\mathcal{F}$ is said to be compact Hausdorff if all leaves are compact and the quotient leaf space $M / \mathcal{F}$ is Hausdorff. The structure of compact Hausdorff foliations has been studied by many authors [10, 12, 30. For a compact Hausdorff foliation $\mathcal{F}$ of a manifold $M$, the transverse holonomy group of each leaf is finite. It follows that the quotient leaf space $M / \mathcal{F}$ is a $V$-manifold in the sense of Satake 37], and the manifold $M$ is a "Seifert fibre space", as introduced by Holmann ([19, cf. [27, 38]), generalizing the classical notion of a Seifert fibration of a 3-manifold 1, 26, 40. In another direction, compact Hausdorff foliations are a special case of compact foliations, where all leaves are assumed compact, but the holonomy groups may be infinite [10, 13, 39, 43, 45].

The goal of this paper is to investigate the properties of the transverse saturated category for compact Hausdorff foliations. When $\mathcal{F}$ is a compact Hausdorff foliation of a compact manifold, $\operatorname{cat}_{\phi}(M)$ is always finite [5, 9]. Our main result, Theorem 6.1 gives estimates from above and below for $\operatorname{cat}_{\phi}(M)$, based on the geometry of the foliation and the topology of the exceptional set $E$. Our examples show that $\operatorname{cat}_{\phi}(M)$ can be arbitrarily large for a fixed quotient space $M / \mathcal{F}$.

The outline of this paper is as follows: In $\S 2$ we give definitions and some properties of transverse category. $\S 3$ recalls the basic properties of compact Hausdorff foliations. $\S 4$ proves that each stratum of the Epstein filtration is a stratified space, and relates the strata to the conjugacy classes of holonomy groups. In $\S 5$ we prove three fundamental propositions used to estimate the transverse category. In $\S 6$ we give our main results and their proofs. Finally, in $\S 7$ we consider the transverse category for some selected examples.

\section{TRAnsverse CATEGORY}

A foliated manifold $M$ will be a connected $C^{\infty}$-manifold, with a $C^{1}$-foliation $\mathcal{F}$ of codimension $q$ and leaf dimension $p$. We will also assume that $M$ is equipped with a Riemannian metric.

Recall that a subset of $X \subset M$ is saturated if it is a union of leaves. Let $\pi: M \rightarrow M / \mathcal{F}$ be the quotient map. Then $X \subset M$ is saturated if and only if $X=\pi^{-1}(\pi(X))$.

By a foliated space, we mean that there are given a foliated manifold $M$ as above, and a saturated subset $X \subset M$ equipped with the restricted foliation $\mathcal{F} \mid X$. A foliation coordinate chart for $\mathcal{F} \mid X$ is the restriction $\varphi: U \cap X \rightarrow \mathbb{D}^{p} \times \mathbb{D}^{q}$ of an

open foliation coordinate chart $\varphi: U \cong \mathbb{D}^{p} \times \mathbb{D}^{q}$ for $\mathcal{F}$. There are more general 
formulations of foliated space (cf. [36, 21, 31, 4]), but for this work we use only this more elementary definition.

An open subset $U \subset M$ is regarded as a foliated manifold with the foliation $\mathcal{F}_{U}$ induced by $\mathcal{F}$. Note that the leaves of $\mathcal{F}_{U}$ are the connected components of the intersections $L \cap U, L$ a leaf of $M$.

Let $(X, \mathcal{F})$ and $\left(X^{\prime}, \mathcal{F}^{\prime}\right)$ be foliated spaces. A homotopy $H: X \times[0,1] \rightarrow X^{\prime}$ is said to be foliated if for all $t \in[0,1]$, the map $H_{t}$ sends each leaf $L$ of $\mathcal{F}$ into another leaf $L^{\prime}$ of $\mathcal{F}^{\prime}$.

An open subset $U$ of $M$ is transversely categorical if there is a foliated homotopy $H: U \times[0,1] \rightarrow M$ such that $H_{0}: U \rightarrow M$ is the inclusion, and $H_{1}: U \rightarrow M$ has image in a single leaf of $\mathcal{F}$. In other words, the open subset $U$ of $M$ is transversely categorical if the inclusion $\left(U, \mathcal{F}_{U}\right) \hookrightarrow(M, \mathcal{F})$ factors through a leaf, up to foliated homotopy.

Definition 2.1. The transverse saturated category $\operatorname{cat}_{巾}(X)$ of a foliated space $(X, \mathcal{F})$ is the least number of transversely categorical, open saturated sets of $M$ required to cover $X$. If no such covering exists, then we set $\operatorname{cat}_{\phi}(X)=\infty$.

When $\mathcal{F}$ is the foliation by points, an open saturated subset is transversely categorical if and only if it is categorical in $M$, so $\operatorname{cat}_{巾}(M)=\operatorname{cat}(M)$. For a foliation defined by a fibration, its transverse saturated category is the category of the leaf (base) space $M / \mathcal{F}$. The transverse category cat ${ }_{\pitchfork}(M)$ is always finite for compact Hausdorff foliations [5, 9] - or see Corollary 5.2 below.

At the other extreme from compact Hausdorff foliations, if $\mathcal{F}$ has a leaf $L_{0}$ which is dense in $M$, then every open saturated set must contain $L_{0}$, hence is also dense. If $M$ is compact, then $\operatorname{cat}_{\pitchfork}(M)=\infty$. There are examples of a foliation $\mathcal{F}$ with noncompact leaves of a compact manifold $M$ with $\operatorname{cat}_{巾}(M)<\infty$; it is even possible for $\mathcal{F}$ to have an exceptional minimal set for $\mathcal{F}$ and still have finite transverse category [20]. It is an open question to classify the foliations for which cat ${ }_{\phi}(M)<\infty$. Here are two partial results.

Theorem $2.2([20])$. If $M$ is a compact manifold and $\operatorname{cat}_{\pitchfork}(M)<\infty$, then $\mathcal{F}$ has a compact leaf.

Theorem $2.3(22])$. If $(M, \mathcal{F})$ is a compact foliation of a compact manifold with $\operatorname{cat}_{\phi}(M)<\infty$, then $\mathcal{F}$ is compact Hausdorff.

The transverse saturated category for Riemannian foliations of compact manifolds has been further studied by the first author $[8$. The results of this paper and the structure theory for Riemannian foliations are applied to give a criterion for when $\operatorname{cat}_{\phi}(M)<\infty$ for a Riemannian foliation.

For the rest of this paper, we consider only the transverse saturated category, and adopt the shorter term transverse category.

\section{Compact Hausdorff foliations}

In this section, we first recall the basic geometric and topological properties of compact Hausdorff foliations. We then give a detailed description of the local geometry of the exceptional set and its natural stratification, introducing concepts and notation that will be used in the proofs in the following sections.

A foliation $\mathcal{F}$ of $M$ is said to be compact if every leaf is a compact submanifold. For example, the orbits of a non-singular flow with every orbit closed defines a 
compact foliation. A fibration $\pi: M \rightarrow B$ of a compact manifold $M$ gives another class of examples of compact foliations, where the fibers of $\pi$ define the leaves of $\mathcal{F}$, with leaf space $M / \mathcal{F}$ naturally diffeomorphic to $B$. In general, the topological space $M / \mathcal{F}$ need not be Hausdorff.

The leaf space $M / \mathcal{F}$ is always a Borel space, so in the sense of measure theory, a compact foliation is type I in the Murray-von Neumann classification [16].

A foliation $\mathcal{F}$ of $M$ is said to be compact Hausdorff if $\mathcal{F}$ is compact, and the leaf space $M / \mathcal{F}$ is a Hausdorff topological space. Compact Hausdorff foliations are equivalent to the class of generalized Seifert fibrations introduced and studied by Holmann [19] and Lee and Raymond [27.

We recall two definitions from 12, 10. First, the Riemannian metric on $T M$ induces a Riemannian metric on each leaf, and hence a leafwise volume form. For $\mathcal{F}$ a compact foliation, the "leaf volume function" vol : $M \rightarrow(0, \infty)$ assigns to $x \in M$ the Riemannian volume of $L_{x}$.

The exceptional set $E$ of a compact foliation $\mathcal{F}$ is the union of all leaves with holonomy. The set of leaves without holonomy, $G=M-E$, is called the good set.

The singularities of the quotient map $\pi: M \rightarrow M / \mathcal{F}$ are concentrated on the exceptional set $E$, as $\pi$ is a fibration on the good set $G$. Millett 30 called the quotient map to the leaf space, $\pi: M \rightarrow M / \mathcal{F}$, a twisted twisted fibration, as $\pi$ is a fibration with "extra twisting" in a neighborhood of $E$.

The first result gives several topological conditions on a compact foliation $\mathcal{F}$ which are equivalent to $M / \mathcal{F}$ being Hausdorff. It summarizes results due to Epstein [12] and Millett [30].

Theorem 3.1 ([12, 30]). Let $\mathcal{F}$ be a compact foliation of a compact manifold $M$. The following conditions are equivalent:

- the holonomy of every leaf is finite;

- there is a bound on the volume of the leaves;

- the quotient space $M / \mathcal{F}$ is Hausdorff;

- $\pi: M \rightarrow M / \mathcal{F}$ is a closed map.

- $\pi: M \rightarrow M / \mathcal{F}$ maps compact sets to closed sets.

Moreover, the volume function is continuous on the good set $G$, while $E$ is the set of points of discontinuity for vol: $M \rightarrow \mathbb{R}$.

Let $\mathbb{D}^{q}$ denote the unit disk in $\mathbb{R}^{q}$ with the Euclidean metric, and $\mathbf{O}(q) \subset \mathbf{G L}\left(\mathbb{R}^{q}\right)$ the subgroup of orthogonal matrices with the standard action on $\mathbb{D}^{q}$. The following is a version for compact Hausdorff foliations of the slice theorems for compact group actions (cf. Chapter IV of [2]). The proof follows from the Reeb structure theorem (34], see also $\S 2$ of [43]) for the neighborhood of a compact leaf with finite holonomy group.

Theorem 3.2. Let $\mathcal{F}$ be a compact Hausdorff foliation of codimension $q$ on a smooth connected manifold $M$. Let $L_{0} \subset G$ be a leaf without holonomy. Given $x \in M$ let $L_{x}$ be the leaf containing $x$.

(1) There are a finite subgroup $H_{x} \subset \mathbf{O}(q)$ and a free action $\alpha_{x}$ of $H_{x}$ on $L_{0}$.

(2) There exists a diffeomorphism of the twisted product

$$
\phi_{x}: L_{0} \times_{H_{x}} \mathbb{D}^{q} \rightarrow V_{x}
$$

onto an open saturated neighborhood $V_{x}$ of $L_{x}$. 
(3) The diffeomorphism $\phi_{x}$ is leaf preserving, where $L_{0} \times_{H_{x}} \mathbb{D}^{q}$ is foliated by the images of $L_{0} \times\{w\}$ for $w \in \mathbb{D}^{q}$ under the quotient map $\mathcal{Q}: L_{0} \times \mathbb{D}^{q} \rightarrow$ $L_{0} \times_{H_{x}} \mathbb{D}^{q}$.

(4) $\phi_{x}$ maps $L_{0} / H_{x} \cong L_{0} \times_{H_{x}}\{0\}$ diffeomorphically to $L_{x}$.

The open set $V_{x}$ is called a standard neighborhood of $L_{x}$, and the 4-tuple $\left(V_{x}, \phi_{x}, H_{x}, \alpha_{x}\right)$ is called a standard local model for $\mathcal{F}$.

For $x \in G$, then $H_{x}$ is trivial and $\phi_{x}$ is a product structure for a neighborhood of $L_{x}$. Hence, the quotient map $\pi: G \rightarrow G / \mathcal{F}$ is a fibration with fibers diffeomorphic to $L_{0}$. In general, for $x \in M$ the leaf $L_{x}$ has an open foliated neighborhood $V_{x}$ as above, and $\phi_{x}: L_{0} \times_{H_{x}} \mathbb{D}^{q} \rightarrow V_{x}$ induces a coordinate map $\hat{\phi}_{b}: \mathbb{D}^{q} / H_{x} \rightarrow W_{b}$, where $W_{b}=\pi\left(V_{x}\right)$. The sets $W_{b} \subset M / \mathcal{F}$ are called basic open sets for $M / \mathcal{F}$, and give $M / \mathcal{F}$ the structure of a Satake manifold [37].

Let $x \in M$ be given, and fix a local model $\left(V_{x}, \phi_{x}, H_{x}, \alpha_{x}\right)$. The following discussion is trivial if $L_{x}$ has no holonomy, where $H_{x}=\{e\}$, so it is the case $x \in E$ that is of interest.

Let $\pi_{x}: L_{0} \times_{H_{x}} \mathbb{D}^{q} \rightarrow L_{0} / H_{x}$ be the map induced from projection onto the first factor. For any leaf $L \subset L_{0} \times_{H_{x}} \mathbb{D}^{q}$ the restriction $\pi_{x}: L \rightarrow L_{x}$ is a covering map. Note that we can use the map $\phi_{x}$ to define a map $Q_{x}=\phi_{x} \circ \pi_{x} \circ \phi_{x}^{-1}$. Then for any leaf $L_{y} \subset V_{x}$ the restriction $Q_{x}: L_{y} \rightarrow L_{x}$ is a covering map. We use the notation $\pi_{x y}=Q_{x} \mid L_{y}$ for the covering map.

Let $x_{0} \in L_{0}$ map to $x$ via the composition $L_{0} \rightarrow L_{0} \times_{H_{x}} \mathbb{D}^{q} \rightarrow V_{x}$. Let $H_{x} \cdot x_{0}$ denote the orbit under $\alpha_{x}$; then the quotient set $\left\{H_{x} \cdot x_{0}\right\} \times_{H_{x}}\{0\}$ is identified with $x$ under $\phi_{x}$.

Define the transversal $\mathcal{D}_{x}$ through $x$

$$
\phi_{x}^{t}: \mathbb{D}^{q} \cong\left\{H_{x} \cdot x_{0}\right\} \times_{H_{x}} \mathbb{D}^{q} \rightarrow \mathcal{D}_{x} \subset V_{x} .
$$

The holonomy of $L_{x}$ with respect to $\mathcal{D}_{x}$ is defined by the path lifting property of $Q_{x}$ (cf. $\S 2$ of Chapter V in [3]), hence is given in the coordinates defined by $\phi_{x}$,

$$
h_{x}: \pi_{1}\left(L_{x}, x\right) \rightarrow H_{x} \subset \mathbf{O}(q) \subset \operatorname{Diffeo}\left(\mathbb{D}^{\mathrm{q}}, 0\right) .
$$

Given $y \in \mathcal{D}_{x}$, the holonomy of the leaf $L_{y}$ containing $y$ is determined by the standard model. Note that there is a unique $w \in \mathbb{D}^{q}$ with $\phi_{x}^{t}(w)=y$. Let $H_{x y}=$ $\left(H_{x}\right)_{w}=\left\{\gamma \in H_{x} \mid \gamma \cdot w=w\right\} \subset H_{x}$ denote the isotropy group at $w \in \mathbb{D}^{q}$ of the linear action of $H_{x}$. Then $\phi_{x}: L_{0} \times_{H_{x y}}\{w\} \rightarrow L_{y}$ is a diffeomorphism, and the covering $Q_{x}: L_{y} \rightarrow L_{x}$ is identified with the map on quotient spaces $\Pi: L_{0} / H_{x y} \rightarrow$ $L_{0} / H_{x}$ induced by the inclusion $H_{x y} \subset H_{x}$ via the commutative diagram

$$
\begin{aligned}
& L_{0} / H_{x y} \stackrel{\cong}{\longrightarrow} L_{0} \times_{H_{x y}}\{w\} \stackrel{\cong \phi_{x}}{\longrightarrow} L_{y} \quad \subset \quad V_{x} \\
& \Pi \downarrow \quad \downarrow \pi_{x} \quad \downarrow \pi_{x y} \quad \downarrow Q_{x} \\
& L_{0} / H_{x} \stackrel{\cong}{\longrightarrow} L_{0} \times_{H_{x}}\{0\} \stackrel{\cong \phi_{x}}{\longrightarrow} L_{x}=L_{x}
\end{aligned}
$$

Let $N_{x y}$ denote the index of the subgroup $H_{x y}$ in $H_{x}$. Then the covering map $\pi_{x y}: L_{y} \rightarrow L_{x}$ has multiplicity $N_{x y}$. We introduce notation for the image of this 
covering map:

$$
\Gamma_{x y}=\text { image }\left\{\left(\pi_{x y}\right)_{\#}: \pi_{1}\left(L_{y}, y\right) \rightarrow \pi_{1}\left(L_{x}, x\right)\right\} \subset \pi_{1}\left(L_{x}, x\right) .
$$

We deduce three properties of the local geometric model for compact Hausdorff foliations.

Lemma 3.3. The holonomy of $L_{y}$ at $y \in \mathcal{D}_{x}$ in the coordinates defined by $\phi_{x}$ is

$$
h_{y}=h_{x} \circ\left(\pi_{x y}\right)_{\#}: \pi_{1}\left(L_{y}, y\right) \rightarrow \pi_{1}\left(L_{x}, x\right) \rightarrow \mathbf{O}(q) .
$$

Hence, $H_{x y}=h_{x}\left(\Gamma_{x y}\right) \subset H_{x}$.

Proof. This is immediate from the discussion above.

Lemma 3.4. Let $y, z \in \mathcal{D}_{x}$ and suppose that $\Gamma_{x y} \subset \Gamma_{x z}$. Then $H_{x y} \subset H_{x z}$, and if $u \in \mathbb{D}^{q}$ with $\phi_{x}^{t}(u)=z$, then $\gamma \cdot u=u$ for all $\gamma \in H_{x y}$.

Proof. $H_{x z}$ is defined as the isotropy group of $u \in \mathbb{D}^{q}$, so $\gamma \in H_{x y} \subset H_{x z}$ fixes $u$.

Let $i_{y}: L_{y} \rightarrow V_{x}$ denote the inclusion map. The above diagram implies that the map on fundamental groups induced by the covering $\pi_{x y}$ is the composition

$$
\left(\pi_{x y}\right)_{\#}=\left(Q_{x}\right)_{\#} \circ\left(i_{y}\right)_{\#}: \pi_{1}\left(L_{y}, y\right) \rightarrow \pi_{1}\left(L_{x}, x\right) .
$$

The following is a key property of foliated homotopy in a compact hausdorff foliation.

Lemma 3.5. Let $H: L_{0} \times[a, b] \rightarrow V_{x}$ be a foliated homotopy, where $L_{0}$ is foliated by the single leaf $L_{0}$. Let $L_{t}$ denote the leaf of $\mathcal{F}$ containing $H_{t}\left(L_{0}\right)$, and set $x_{t}=H_{t}\left(x_{0}\right)$. Suppose that

$$
\left(H_{t}\right)_{\#}: \pi_{1}\left(L_{0}, x_{0}\right) \rightarrow \pi_{1}\left(L_{t}, x_{t}\right)
$$

is injective for $t=a$. Then $\left(H_{t}\right)_{\#}$ is injective for all $a \leq t \leq b$.

Proof. The composition

$$
\tau^{*} \circ\left(Q_{x} \circ i_{t} \circ H_{t}\right)_{\#}: \pi_{1}\left(L_{0}, x_{0}\right) \rightarrow \pi_{1}\left(L_{x}, Q_{x}\left(x_{t}\right)\right) \cong \pi_{1}\left(L_{x}, x\right)
$$

is injective for $t=a$, where the isomorphism $\tau^{*}$ is induced by the path $\tau(t)=$ $Q_{x}\left(x_{t}\right)$. As $\tau^{*} \circ\left(Q_{x} \circ i_{t} \circ H_{t}\right)_{\#}$ is constant under homotopy, the composition remains injective for all $t$; hence $\left(H_{t}\right)_{\#}$ is injective for all $t$.

\section{EPSTEIN FILTRATION}

The Epstein filtration 11, 12, 10, 13 of the exceptional set $E$ is a descending countable chain of closed saturated subsets of $E$, each level defined inductively in terms of the restricted holonomy of the foliation in the preceding level. This chain of subsets need not have finite length; Vogt [45] has constructed examples of compact foliations of compact manifolds for which the filtration has length equal to any specified countable ordinal. For compact Hausdorff foliations, the Epstein filtration has finite length, and closed subsets in the chain have local geometric descriptions, all in terms of the isotropy groups of the local holonomy actions. In this section, we discuss the local structure of the Epstein filtration of a compact Hausdorff foliation in detail, as it is central to our analysis of foliated homotopies in the next section. 
Set $E^{0}=M$, and define the first level of the Epstein filtration by $E^{1}=E$. (Note that some authors use the alternate convention $E^{0}=E$.) Assume that $E^{\ell}$ has been defined; then $E^{\ell+1} \subset E^{\ell}$ is the union of the leaves in $E^{\ell}$ with holonomy for the restricted foliation $\mathcal{F} \mid E^{\ell}$ on $E^{\ell}$. That is, $x \in E^{\ell}$ belongs to $E^{\ell+1}$ if

(1) there is a holonomy map $h: \mathcal{D}_{x} \rightarrow \mathcal{D}_{x}$ of $L_{x}$ which leaves the set $E^{\ell} \cap \mathcal{D}_{x}$ invariant, and

(2) the restriction $h \mid E^{\ell}: E^{\ell} \rightarrow E^{\ell}$ defines a non-trivial element of the holonomy group for $\mathcal{F} \mid E^{\ell}$.

We introduce two basic concepts before stating the main result of this section.

Definition 4.1. A closed subset $X \subset M$ is a stratified space if for each $x \in X$ there exists an open coordinate neighborhood $\varphi: U \rightarrow \mathbb{D}^{p+q}$ of $x$ such that $U \cap X$ maps to a finite union of linear spaces through the origin in $\mathbb{D}^{p+q}$.

For a subset $\mathcal{B} \subset \mathbf{O}(q)$, let $\langle\mathcal{B}\rangle \subset \mathbf{O}(q)$ denote the subgroup generated by the elements of $\mathcal{B}$. For a single element $g$ let $\langle g\rangle$ be the cyclic subgroup it generates. The identity element is $e \in \mathbf{O}(q)$.

Given a subset $\mathcal{B} \subset \mathbf{O}(q)$, let $W(\mathcal{B}) \subset \mathbb{D}^{q}$ be the fixed-point set for the subgroup $\langle\mathcal{B}\rangle$ acting on $\mathbb{D}^{q}$. For $\mathcal{B}=\emptyset$ set $W(\emptyset)=\mathbb{D}^{q}$. Let $W(g)$ denote the subspace fixed by the cyclic subgroup $\langle g\rangle$. Then

$$
W(\mathcal{B})=\bigcap_{g \in \mathcal{B}} W(g)
$$

$W(\mathcal{B})$ is a proper linear subspace if $\mathcal{B} \neq\{e\}$. If $W(\mathcal{B}) \subset \mathbb{D}^{q}$ has codimension one, then $\mathcal{B}$ must consist of a single orientation-reversing isometry; otherwise, $W(\mathcal{B})$ has codimension at least two.

Definition 4.2. A subset $\mathcal{B} \subset \mathbf{O}(q)$ is said to be $\ell$-regular if there exist $\left\{g_{1}, \ldots, g_{\ell}\right\}$ $\subset\langle\mathcal{B}\rangle$ such that for all $1 \leq i \leq \ell$,

$$
W\left(\left\langle g_{1}, \ldots, g_{i}\right\rangle\right) \subset W\left(\left\langle g_{1}, \ldots, g_{i-1}\right\rangle\right)
$$

is a proper subspace, where $W\left(\left\langle g_{1}, \ldots, g_{i-1}\right\rangle\right)=\mathbb{D}^{q}$ for $i=1$. In particular, this implies that $W\left(g_{i}\right) \neq \mathbb{D}^{q}$ for all $i$. The set $\left\{g_{1}, \ldots, g_{\ell}\right\} \subset\langle\mathcal{B}\rangle$ is called an $\ell$-regular sequence.

Condition (44) is equivalent to requiring that the action of $g_{i}$ on the subspace $W\left(\left\langle g_{1}, \ldots, g_{i-1}\right\rangle\right)$ is not the identity. It does not assume, however, that $g_{i}$ maps this subspace to itself.

Note that the property of being a regular sequence is invariant under conjugation in $\mathbf{O}(q)$.

We use the notation $\left[g_{1} \cdots g_{\ell}\right]$ to indicate that $\left\{g_{1}, \ldots, g_{\ell}\right\}$ is an $\ell$-regular sequence.

Proposition 4.3. Let $\mathcal{F}$ be a compact Hausdorff foliation with exceptional set $E$. For each $\ell>0$, the stratum $E^{\ell}$ of the Epstein filtration is a stratified space. Moreover, $y \in E^{\ell}$ if and only if for any standard local model $\left(V_{x}, \phi_{x}, H_{x}, \alpha_{x}\right)$ with $y \in V_{x}$ the holonomy $H_{x y}$ is $\ell$-regular.

Proof. For $x \in E^{\ell}$, let $\left(V_{x}, \phi_{x}, H_{x}, \alpha_{x}\right)$ be a standard local model near $L_{x}$. We show that the intersection $E^{\ell} \cap V_{x}$ is a finite union of submanifolds of $V_{x}$, each defined by an $\ell$-regular sequence. 
We first consider the case $\ell=1$. Define the union of linear spaces

$$
W_{x}^{1}=\bigcup_{[g] \subset H_{x}} W(g),
$$

where the union over $[g] \subset H_{x}$ is the union over all $g \neq e$. Note that $W_{x}^{1}$ is invariant under $H_{x}$. The key point is that $w \in W_{x}^{1}$ exactly when $\left(H_{x}\right)_{w}=\left\{\gamma \in H_{x} \mid \gamma \cdot w=\right.$ $w\}$ is non-trivial. Hence,

$$
E \cap V_{x}=\phi_{x}\left(L_{0} \times_{H_{x}} W_{x}^{1}\right) .
$$

Moreover, $w \in W_{x}(g)$ if and only if $g \in\left(H_{x}\right)_{w}$, so for $y=\phi_{x}^{t}(w)$,

$$
\langle g\rangle \subset H_{x y} \Longleftrightarrow y \in \phi_{x}^{t}(W(g)) .
$$

Combining (6) and (7), we obtain for $x \in W(g)$

$$
y=\phi_{x}^{t}(w) \in E \cap V_{x} \Longleftrightarrow g \subset H_{x y} \Longleftrightarrow H_{x y} \text { is 1-regular. }
$$

This implies that $E \cap V_{x}$ is the union of the images of the hyperplanes $W(g)$ for $g \neq e$, and so $E$ is a stratified space as in Definition 4.1

The description of $E^{\ell}$ for $\ell>1$ in terms of regular sequences is analogous. Define

$$
W_{x}^{\ell}=\bigcup_{\left[g_{1} \cdots g_{\ell}\right] \subset H_{x}} W\left(\left\langle g_{1}, \ldots, g_{\ell}\right\rangle\right) .
$$

Note that $W_{x}^{\ell}$ is invariant under the action of $H_{x}$. We show that $E^{\ell} \cap V_{x}=$ $\phi_{x}\left(L_{0} \times_{H_{x}} W_{x}^{\ell}\right)$. Suppose that $y \in E^{\ell} \cap \mathcal{D}_{x}$ and let $w=\left(\phi_{x}^{t}\right)^{-1}(y)$. By induction,

$$
E^{\ell-1} \cap V_{x}=\phi_{x}\left(L_{0} \times_{H_{x}} W_{x}^{\ell-1}\right) .
$$

As $y \in E^{\ell-1} \cap \mathcal{D}_{x}$, there exists an $(\ell-1)$-regular sequence $\left\{g_{1}, \ldots, g_{\ell-1}\right\} \subset H_{x y}$ for which $w \in W\left(\left\langle g_{1} \cdots g_{\ell-1}\right\rangle\right)$.

Since $y \in E^{\ell}$, there exists some $g_{\ell} \in H_{x y}$ whose restricted action on $W_{x}^{\ell-1}$ is non-trivial in an open neighborhood of $y$. It follows that there exists an $(\ell-1)$ regular sequence $\left\{g_{1}, \ldots, g_{\ell-1}\right\} \subset H_{x y}$ such that both $w \in W\left(\left\langle g_{1} \cdots g_{\ell-1}\right\rangle\right)$ and $g$ does not restrict to the identity on $W\left(\left\langle g_{1} \cdots g_{\ell-1}\right\rangle\right)$. Hence, $\left\{g_{1}, \ldots, g_{\ell-1}, g_{\ell}\right\}$ is an $\ell$-regular sequence.

This shows $E^{\ell} \cap \mathcal{D}_{x} \subset \phi_{x}\left(L_{0} \times_{H_{x}} W_{x}^{\ell}\right)$, and hence $E^{\ell} \cap V_{x} \subset \phi_{x}\left(L_{0} \times_{H_{x}} W_{x}^{\ell}\right)$. The converse inclusion is immediate from the definition of the set $W_{x}^{\ell}$, so we have

$$
E^{\ell} \cap V_{x}=\phi_{x}\left(L_{0} \times_{H_{x}} W_{x}^{\ell}\right) .
$$

In particular, this implies that $y \in E^{\ell} \cap \mathcal{D}_{x} \Longleftrightarrow H_{x y}$ is $\ell$-regular. of

Note that $W_{x}^{\ell}$ is a union of linear subspaces of $\mathbb{D}^{q}$. For $\left[g_{1} \ldots g_{\ell}\right] \subset H_{x}$ the image

$$
L_{0} \times W\left(\left\langle g_{1} \ldots g_{\ell}\right\rangle\right) \rightarrow L_{0} \times_{H_{x}} W_{x}^{\ell} \rightarrow E^{\ell} \cap V_{x}
$$

is a submanifold, and $E^{\ell} \cap V_{x}$ is the union of these images. Thus, $E^{\ell}$ is a stratified space.

We observe several corollaries which follow from the above proof.

Corollary 4.4. Let $\mathcal{F}$ be a compact Hausdorff foliation of a compact manifold $M$, and $X \subset E^{\ell}-E^{\ell+1}$ a connected component. Then $X$ is a submanifold of $M$. 
Proof. Let $X \subset E^{\ell}-E^{\ell+1}$ be a connected component, and let $\left(V_{x}, \phi_{x}, H_{x}, \alpha_{x}\right)$ be a standard local model near $L_{x}$ for $x \in X$. Recall that equation (10) gives a local structure, $E^{\ell} \cap V_{x}=\phi_{x}\left(L_{0} \times_{H_{x}} W_{x}^{\ell}\right)$, where

$$
W_{x}^{\ell}=\bigcup_{\left[g_{1} \cdots g_{\ell}\right] \subset H_{x}} W\left(\left\langle g_{1}, \ldots, g_{\ell}\right\rangle\right)
$$

and each $W\left(\left\langle g_{1}, \ldots, g_{\ell}\right\rangle\right)$ is a linear subspace of $\mathbb{D}^{q}$. If there are $\ell$-regular sequences $\left[g_{1} \cdots g_{\ell}\right]$ and $\left[h_{1} \cdots h_{\ell}\right]$ with $W\left(\left\langle g_{1}, \ldots, g_{\ell}\right\rangle\right) \neq W\left(\left\langle h_{1}, \ldots, h_{\ell}\right\rangle\right)$, then either some element in $\left\{h_{1}, \ldots, h_{k}\right\} \subset H_{x}$ has non-trivial restriction to $W\left(\left\langle g_{1}, \ldots, g_{\ell}\right\rangle\right)$, or one of $\left\{g_{1}, \ldots, g_{\ell}\right\} \subset H_{x}$ has non-trivial restriction to $W\left(\left\langle h_{1}, \ldots, h_{k}\right\rangle\right)$. In either case, $H_{x}$ admits an $\ell+1$ regular sequence, so $x \in E^{\ell+1}$, contrary to hypothesis. It follows that $W_{x}^{\ell}$ is a linear subspace of $\mathbb{D}^{q}$; hence $L_{0} \times{ }_{H_{x}} W_{x}^{\ell}$ is a non-singular manifold, and $\phi_{x}$ defines coordinate charts for the points in $X \cap V_{x}$.

Corollary 4.5. Let $\mathcal{F}$ be a compact Hausdorff foliation of a compact manifold $M$. Then there exists $N$ such that for any $x \in E$ and any standard local model $\left(V_{x}, \phi_{x}, H_{x}, \alpha_{x}\right)$, the intersection $E^{\ell} \cap V_{x}$ is the union of the images of at most $N$ subspaces $W\left(\left\langle g_{1}, \ldots, g_{i}\right\rangle\right) \subset \mathbb{D}^{q}$. Hence, each $E^{\ell}$ is relatively closed and nowhere dense in $M$.

Proof. For $x \in E^{\ell}$, let $\left(V_{x}, \phi_{x}, H_{x}, \alpha_{x}\right)$ be a standard local model near $L_{x}$. As $H_{x}$ is a finite group, there are a finite number of $\ell$-regular sequences $\left\{g_{1} \ldots g_{\ell}\right\} \subset H_{x}$. Hence, the intersection $E^{\ell} \cap V_{x}$ is a finite union of at most $N_{x}$ submanifolds of $V_{x}$, each defined by the image of the fixed-point set $W\left(\left\langle g_{1} \ldots g_{\ell}\right\rangle\right) \subset \mathbb{D}^{q}$ for some regular sequence. The compact manifold $M$ has a finite covering by standard local models, centered at points $\left\{x_{1}, \ldots, x_{d}\right\}$. Then take $N=\max \left\{N_{x_{1}}, \ldots, N_{x_{d}}\right\}$.

Corollary 4.6. Let $\mathcal{F}$ be a compact Hausdorff foliation with exceptional set E. Then the Epstein filtration has finite length $\nu=\nu(\mathcal{F}) \leq q$,

$$
\emptyset=E^{\nu+1} \subset E^{\nu} \subset E^{\nu-1} \subset \cdots \subset E^{1}=E .
$$

Proof. For $x \in E^{\ell}$, let $\left(V_{x}, \phi_{x}, H_{x}, \alpha_{x}\right)$ be a standard local model near $L_{x}$. The intersection $E^{\ell} \cap V_{x}$ is a finite union of submanifolds of $V_{x}$, each defined by the image of the fixed-point set $W\left(\left\langle g_{1} \ldots g_{\ell}\right\rangle\right) \subset \mathbb{D}^{q}$, where $\left\{g_{1} \ldots g_{\ell}\right\}$ is an $\ell$-regular sequence. By definition, for each $1 \leq i \leq \ell, W\left(\left\langle g_{1}, \ldots, g_{i}\right\rangle\right) \subset W\left(\left\langle g_{1}, \ldots, g_{i-1}\right\rangle\right)$ is a proper subspace. Thus $\ell \leq q$.

Corollary 4.7. Let $\mathcal{F}$ be a compact Hausdorff foliation with exceptional set $E$. If $\mathcal{F}$ has orientable normal bundle, then the good set $G$ is open, dense, and locally path connected.

Proof. The fixed-point set of an orientation-preserving isometry has codimension at least 2; hence each $W\left(\left\langle g_{1} \ldots g_{\ell}\right\rangle\right) \subset \mathbb{D}^{q}$ has codimension at least 2 in $\mathbb{D}^{q}$. Thus for each local model $\left(V_{x}, \phi_{x}, H_{x}, \alpha_{x}\right)$, the complement of $E \cap V_{x}$ in $V_{x}$ is open, dense, and locally path connected.

There are several alternate approaches to defining a filtration of the exceptional set $E$ of a compact Hausdorff foliation. These can be related to the Epstein filtration discussed above, and are of interest in part due to the connections with the study of LS-category for finite group actions. For example, Haefliger described in [17], page 80 , a partition of $E$ by conjugacy classes of the holonomy groups. This partition of $E$ is analogous to the partition of the singular set of a group action by the conjugacy 
classes of the stabilizers (cf. also [32] and $\S \S 5,6$ of Chapter 1 in 41]). We briefly discuss Haefliger's approach, before continuing with the homotopy lemmas in the next section.

Given $y \in M$, choose a framing of the normal bundle to $\mathcal{F}$ at $y$, and let $\mathcal{H}_{y} \subset$ $\mathbf{G L}\left(\mathbb{R}^{q}\right)$ denote the linear holonomy group of the leaf $L_{y}$ though $y$ with respect to this framing. The conjugacy class $\left\{\mathcal{H}_{y}\right\}$ of the holonomy group is independent of the choice of framing, so $y \mapsto\left\{H_{y}\right\}$ is a well-defined function on $M$. Moreover, if $z \in L_{y}$, then $\left\{H_{y}\right\}=\left\{H_{z}\right\}$, so the conjugacy class function $y \mapsto\left\{H_{y}\right\}$ is constant on leaves.

We assume $\mathcal{F}$ is a compact Hausdorff foliation. Define an equivalence relation on $M$ where $x \sim_{h} y$ if $\left\{\mathcal{H}_{x}\right\}=\left\{\mathcal{H}_{y}\right\}$. The connected components of the equivalence classes then define a partition of $M$ by saturated sets. We denote the components of this partition by $\left\{X_{\alpha} \mid \alpha \in \mathcal{A}\right\}$.

For example, all leaves with trivial holonomy form one equivalence class, which is just the good set $G$ of $\mathcal{F}$. If $G$ is connected, then $G=X_{\alpha}$ for some $\alpha$. Otherwise, the good set is partitioned into its connected components, i.e., a union of sets $X_{\alpha_{1}} \cup \cdots \cup X_{\alpha_{r}}$. At the other extreme, if $E^{\nu}$ is the lowest non-empty level of the Epstein filtration, then $E^{\nu}$ is closed, and $\left\{\mathcal{H}_{y}\right\}$ is constant on each connected component $E^{\nu}$, so again $E^{\nu}=X_{\beta_{1}} \cup \cdots \cup X_{\beta_{s}}$ for some indices $\beta_{1}, \ldots, \beta_{s}$.

These two cases illustrate the general relationship between the Epstein filtration and the partition of $M$ by $\sim_{h}$ :

Proposition 4.8. The sets $\left\{X_{\alpha} \mid \alpha \in \mathcal{A}\right\}$ are exactly the connected components of the strata $E^{\ell}-E^{\ell+1}$ of the Epstein filtration.

Proof. Let $X \subset E^{\ell}-E^{\ell+1}$ be a connected component, and let $\left(V_{x}, \phi_{x}, H_{x}, \alpha_{x}\right)$ be a standard local model near $L_{x}$ for $x \in X$. Recall that equation (10) gives a local structure, $E^{\ell} \cap V_{x}=\phi_{x}\left(L_{0} \times_{H_{x}} W_{x}^{\ell}\right)$, and by the proof of Corollary 4.4. $W_{x}^{\ell}$ is a linear subspace of $\mathbb{D}^{q}$. In particular, for all $y \in W_{x}^{\ell}, H_{x y}=H_{x}$. Thus $\left\{H_{y}\right\}=\left\{H_{x}\right\}$, and so the conjugacy class function $\left\{H_{y}\right\}$ is constant on $X \cap V_{x}$. As $X$ is connected, this implies $\left\{H_{y}\right\}$ is constant on $X$, so $X \subset X_{\alpha}$ for some $\alpha \in \mathcal{A}$

Conversely, let $X_{\alpha}$ be a connected saturated set such that $\left\{H_{x}\right\}$ is constant for $x \in X_{\alpha}$.

If $\left\{H_{x}\right\}$ is trivial, then $X_{\alpha} \subset G=E^{0}$. Moreover, $X \cap E^{1}=\emptyset$; if not, then there is $x \in X \cap E^{1}$ and so $H_{x}$ must have a 1-regular sequence. In particular, $\left\{H_{x}\right\}$ cannot be trivial, contrary to hypothesis.

In general, let $\ell>0$ be the greatest integer for which $X_{\alpha} \subset E^{\ell}$, so $X \not \subset E^{\ell+1}$. We claim that $X \cap E^{\ell+1}=\emptyset$; if not, then there is $x \in X \cap E^{\ell+1}$ and so $H_{x}$ must have an $\ell+1$-regular sequence. For all $y \in X$ we are given that $\left\{H_{y}\right\}=\left\{H_{x}\right\}$, so $\left\{H_{y}\right\}$ also has an $\ell+1$-regular sequence. By Proposition 4.3, this implies $X \subset E^{\ell+1}$, contrary to hypothesis. Thus, $X$ is a connected subset of $E^{\ell}-E^{\ell+1}$ and hence is contained in one of its connected components.

The decomposition of $M$ into sets $\left\{X_{\alpha} \mid \alpha \in \mathcal{A}\right\}$ can be used to define a new filtration of $M$. Let $\mathcal{E}_{0}$ be the union of all connected components of the equivalence classes which are closed in $M$. Assume that $\mathcal{E}_{\ell-1}$ has been defined; then $\mathcal{E}_{\ell}$ is the union of $\mathcal{E}_{\ell-1}$ with all connected components of the equivalence classes whose boundaries are contained in $\mathcal{E}_{\ell-1}$.

This filtration of $M$ is closely related to the Epstein filtration, for if $\mathcal{E}_{\ell} \subset E^{k}$, then $\mathcal{E}_{\ell+1} \subset E^{k-1}$. Furthermore, if each level $\mathcal{E}_{\ell}$ is connected, then it is not hard 
to show that $\mathcal{E}_{\ell}=E^{\nu-\ell}$ for $0 \leq \ell \leq \nu$. However, in the general case, the inclusions need not follow such a simple incremental rule.

There is yet another approach to the stratification of the set $E$, which is essentially local, and related to standard methods in the theory of finite group actions. For a standard local model $\left(V_{x}, \phi_{x}, H_{x}, \alpha_{x}\right)$, consider the subgroups of the holonomy group $H_{x}$ with the partial ordering by inclusion. Associate to $\Lambda \subset H_{x}$ the set of points $V_{\Lambda}=\left\{y \in V_{x} \mid H_{x y}=\Lambda\right\}$. If $\Lambda \subset \Lambda^{\prime}$ then $V_{\Lambda^{\prime}} \subset V_{\Lambda}$, so we obtain a partial ordering of the sets $V_{\Lambda}$ for $\Lambda \subset H_{x}$. Millett proposed a filtration for compact Hausdorff foliations based on these ideas in [30]. Note that the union of the sets $V_{\Lambda}$ over the conjugacy classes of $\Lambda$ is contained in one of the sets $\mathcal{E}_{\ell} \cap V_{x}$.

The stratification of $E \cap V_{x}$ by the partial ordering of the subsets of the local holonomy is clearly local - to extend this to a stratification of $E$ requires a hypothesis of compatibility between the local strata. For example, given two standard open sets $V_{x}$ and $V_{y}$ with $V_{x} \cap V_{y} \neq \emptyset$, a subgroup $\Lambda \subset H_{x}$ determines a priori only a conjugacy class of groups $\{\Lambda\}$ in $H_{y}$. What is needed is to give a "natural" correspondence between subgroups $\Lambda \subset H_{x}$ and $\Lambda^{\prime} \subset H_{y}$ so that the partial ordering by inclusion is preserved. One way to achieve this is to assume there is a global finite group action on a topological space, whose restrictions yield the local actions of the $H_{x}$. Related to this problem, Haefliger formulated a general concept of graph of groups in [18; the transverse structure of a compact Hausdorff foliation provides an important class of examples. Haefliger's theory provides a framework for studying how the local models of a compact Hausdorff foliation are globally assembled, which may lead to another approach to stratifying the exceptional set $E$ (cf. also Chapter IV of [2], and [33]).

These discussions are relevant to the theme of this paper, which is that foliated homotopies preserve the Epstein filtration, and this implies estimates on the transverse category in terms of the geometry of $\mathcal{F}$. There are special cases of compact Hausdorff foliations where foliated homotopy also preserves some of the additional filtrations above. One such special case is when $\mathcal{F}$ is induced from the action of a finite group $G$ on a compact manifold $X$. Another is when $\mathcal{F}$ is defined by the action of a compact Lie group $G$ acting on the manifold $M$. Colman has studied the $G$-category theory in these cases [7]. In particular, her work gives examples where the various filtrations differ, and moreover yield different estimates for the $G$-category.

\section{Foliated homotopies}

A basic point of this paper is that a foliated homotopy of a saturated set in a compact Hausdorff foliation preserves certain transverse geometric properties of the foliation. For example, it is relatively easy to prove that the exceptional set is preserved under foliated homotopy. The exceptional set itself has the structure of a stratified space, and each stratum is preserved by a foliated homotopy.

In this section we establish three fundamental results used to obtain estimates on the transverse category of compact Hausdorff foliations. The first proposition extends a basic result of the first author [5, 9.

Theorem 5.1 (Basic). Let $\mathcal{F}$ be a compact Hausdorff foliation, $x \in M$, and $\left(V_{x}, \phi_{x}, H_{x}, \alpha_{x}\right)$ a standard local model for $\mathcal{F}$. Then $V_{x}$ is transversely categorical. Moreover, for each stratum $E^{\ell}$ of the exceptional set $E$ the relatively open 
subset $V_{x} \cap E^{\ell}$ of $E^{\ell}$ is transversely categorical in $E^{\ell}$ for the restricted foliation $\mathcal{F} \mid E^{\ell}$.

Proof. The radial contraction map $R(t): \mathbb{D}^{q} \rightarrow \mathbb{D}^{q}$ defined by multiplying the points of $\mathbb{D}^{q}$ by $t$ commutes with the action of $\mathbf{O}(q)$, so it induces a transverse contraction map, also denoted

$$
R(t): L_{0} \times_{H_{x}} \mathbb{D}^{q} \rightarrow L_{0} \times_{H_{x}} \mathbb{D}^{q} .
$$

We define a foliated homotopy by

$$
H_{t}=\phi_{x} \circ R(1-t) \circ \phi_{i}^{-1}: V_{x} \rightarrow V_{x},
$$

where $H_{0}=I d$ and $H_{1}$ has image in $L_{x}$.

For $x \in E^{\ell}$, let $W_{x}^{i}=\left(\phi_{x}^{t}\right)^{-1}\left(E^{\ell} \cap \mathcal{D}_{x}\right) \subset \mathbb{D}^{q}$ be the set of points corresponding to leaves of $E^{\ell} \cap \mathcal{D}_{x}$. Then $W_{x}^{i}$ consists of a finite union of planes through $0 \in \mathbb{R}^{q}$ intersected with $\mathbb{D}^{q}$, hence is invariant under the radial contraction map $R(t)$. The restriction of $H_{t}$ to $E^{\ell}$ thus preserves $E^{\ell}$.

Corollary 5.2. Let $\mathcal{F}$ be a compact Hausdorff foliation of a compact manifold $M$. Then $\operatorname{cat}_{\pitchfork}(M)<\infty$. Moreover, for each stratum $E^{\ell} \subset E$, cat $_{\pitchfork}\left(E^{\ell}\right)<\infty$.

Proof. Each point $x \in M$ has a standard neighborhood $\left(V_{x}, \phi_{x}, H_{x}, \alpha_{x}\right)$. Choose a finite subcovering of $M$ by the saturated open sets $\left\{V_{x_{1}}, \ldots, V_{x_{n}}\right\}$. By Theorem 5.1 each set $V_{x_{i}}$ is transversely categorical; hence $\operatorname{cat}_{巾}(M) \leq n$. Similarly, for a stratum $E^{\ell}$, the collection $\left\{V_{y} \mid y \in E^{\ell}\right\}$ is an open covering by saturated open sets, and $E^{\ell}$ compact implies there is a finite subcovering $\left\{V_{y_{1}}, \ldots, V_{y_{m}}\right\}$. Again by Theorem 5.1, each relatively open set $V_{y_{i}} \cap E^{\ell}$ is transversely categorical; hence $\operatorname{cat}_{\phi}\left(E^{\ell}\right) \leq m$.

The second result is one of the fundamental observations of this paper. It shows that the presence of the exceptional set $E$ imposes restrictions on the transversely categorical open saturated sets.

Theorem 5.3 (Homotopy). Let $X \subset M$ be a saturated set, and let $H: X \times[0,1] \rightarrow$ $M$ be a foliated homotopy, where $H_{0}$ is the inclusion. Then for all $1 \leq \ell \leq \nu$ and all $0 \leq t \leq 1$ we have $H_{t}\left(X \cap E^{\ell}\right) \subset E^{\ell}$. That is, a foliated homotopy preserves the Epstein filtration.

Proof. Let $X \subset M$ be a saturated set, and $H: X \times[0,1] \rightarrow M$ a foliated homotopy, with $H_{0}$ the inclusion. Let $L_{0} \subset E^{\ell} \cap X$ for some $1 \leq \ell \leq \nu$. As $H$ is a foliated homotopy, each image $L_{t}^{\prime}=H_{t}\left(L_{0}\right)$ lies in a leaf $L_{t}$ of $\mathcal{F}$. We show that $L_{t} \subset E^{\ell}$ for all $t$.

Pick a basepoint $x_{0} \in L_{0}$ and define $x_{t}=H_{t}\left(x_{0}\right)$ for $0 \leq t \leq 1$. Let $\sigma(t)=x_{t}$ denote the continuous path determined by the $x_{t}$.

Cover the image $\sigma[0,1]$ with a finite set of standard neighborhoods $\left\{V_{x_{0}}, V_{x_{1}}, \ldots\right.$, $\left.V_{x_{n}}\right\}$, where $x_{i}=\sigma\left(t_{i}\right)$ with $0=t_{0}<t_{1}<\cdots<t_{n}=1$. We require that the partition be chosen small enough so that for each $0 \leq i<n$ we have $\sigma\left(\left[t_{i-1}, t_{i+1}\right]\right) \subset$ $V_{x_{i}}$, where $t_{-1}=0$.

In the following, we adopt the notational convention replacing the subscript $x_{i}$ with the index $i$ when there is no chance of confusion. For example, $V_{i}=V_{x_{i}}$, $L_{i}=L_{x_{i}}$ and $\mathcal{D}_{i}=\mathcal{D}_{x_{i}}$.

Figure 1 gives a sketch of the initial neighborhood $V_{x_{0}}$ and the path $\sigma$. 


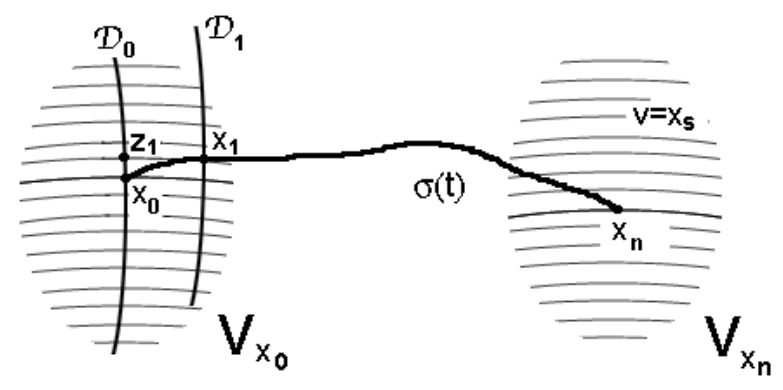

FiguRE 1.

We use the standard local neighborhoods to project the restricted path

$$
\sigma:\left[t_{i-1}, t_{i+1}\right] \rightarrow V_{i}
$$

to the local transversal $\mathcal{D}_{i}$. For each $i$, there is a path $z_{i}(t) \in \mathcal{D}_{i}$ for $t_{i-1} \leq t \leq t_{i+1}$, the path traced out in the transversal through $x_{i}$ by the intersection with the leaves $L_{t}$ starting with $z_{i}\left(t_{i}\right)=x_{i}$. Set $z_{i+1}=z_{i}\left(t_{i+1}\right) \in \mathcal{D}_{i}$.

Also, compose the path $\sigma$ with the projection $Q_{x_{i+1}}$ to the leaf $L_{i+1}$ to obtain a leafwise path $\tau_{i}=Q_{x_{i+1}} \circ \sigma:\left[t_{i}, t_{i+1}\right] \rightarrow L_{i+1}$ with $\tau_{i}\left(t_{i}\right)=z_{i+1}$ and $\tau_{i}\left(t_{i+1}\right)=x_{i+1}$. This induces an isomorphism

$$
\tau_{i}^{*}: \pi_{1}\left(L_{i+1}, z_{i+1}\right) \cong \pi_{1}\left(L_{i+1}, x_{i+1}\right) .
$$

The holonomy of $\mathcal{F}$ along $\tau_{i}$ induces a local diffeomorphism $h_{\tau_{i}}$ between a neighborhood of $z_{i+1} \in \mathcal{D}_{i}$ and a neighborhood of $x_{i+1} \in \mathcal{D}_{i+1}$. The map $h_{\tau_{i}}$ conjugates the holonomy group $\mathcal{H}_{z_{i+1}}$ of $L_{i+1}$ based at $z_{i+1}$ to the holonomy group $\mathcal{H}_{x_{i+1}}$ of $L_{i+1}$ based at $x_{i+1}$, so that the following diagram commutes:

$$
\begin{array}{cll}
\pi_{1}\left(L_{i+1}, z_{i+1}\right) & \stackrel{h_{z_{i+1}}}{\longrightarrow} & \mathcal{H}_{z_{i+1}} \\
\tau_{i}^{*} \quad \downarrow & \downarrow h_{\tau_{i}} \\
\pi_{1}\left(L_{i+1}, x_{i+1}\right) & \stackrel{h_{x_{i+1}}}{\longrightarrow} & \mathcal{H}_{x_{i+1}}
\end{array}
$$

After this geometric set-up, the proof proceeds by induction on the index $i$ of the neighborhoods, and the results of sections 3 and 4 .

The initial map $H_{0}$ is an inclusion, so by Lemma 3.5 the maps $\left(H_{t}\right)_{\#}: \pi_{1}\left(L_{0}, x_{0}\right)$ $\rightarrow \pi_{1}\left(L_{t}, x_{t}\right)$ are injective for $0 \leq t \leq t_{1}$. Lemma 3.3 implies the isotropy group $H_{x_{0}} \subset H_{x_{0} z_{0}(t)}$ for $0 \leq t \leq t_{1}$. By Proposition $4.3 x_{0} \in E^{\ell}$ implies there exists an $\ell$-regular sequence $\left\{g_{1}, \ldots, g_{\ell}\right\} \subset H_{x_{0}}$; hence $\left\{g_{1}, \ldots, g_{\ell}\right\}$ is an $\ell$-regular sequence in $H_{x_{0} z_{0}(t)}$. Thus, $z_{0}(t) \in E^{\ell} \cap \mathcal{D}_{0}$ for $0 \leq t \leq t_{1}$. As $H_{t}$ is a foliated map, this implies $H_{t}\left(L_{0}\right) \subset E^{\ell}$ for $0 \leq t \leq t_{1}$. By the commutative diagram (11) for $i=0$, the holonomy group $H_{x_{1}}$ contains an $\ell$-regular sequence $\left\{h_{1}, \ldots, h_{\ell}\right\}$ in the image of

$$
h_{x_{1}} \circ\left(H_{t_{1}}\right)_{\#}: \pi_{1}\left(L_{0}, x_{0}\right) \rightarrow \pi_{1}\left(L_{x_{1}}, x_{1}\right) \rightarrow \mathbf{O}(q) .
$$

Now proceed inductively on $i \geq 1$, where the argument above carries over exactly the same. Assume that for $0 \leq t \leq t_{i}$ the leaf $H_{t}\left(L_{0}\right) \subset E^{\ell}$, the induced map on 
fundamental groups $\left(H_{t}\right)_{\#}: \pi_{1}\left(L_{0}, x_{0}\right) \rightarrow \pi_{1}\left(L_{t}, x_{t}\right)$ is injective, and there is an $\ell$-regular sequence in the image of

$$
h_{x_{i}} \circ\left(H_{t_{i}}\right)_{\#}: \pi_{1}\left(L_{0}, x_{0}\right) \rightarrow \pi_{1}\left(L_{x_{i}}, x_{i}\right) \rightarrow \mathbf{O}(q) .
$$

This is shown above for $i=1$.

Lemma 3.5 applied to $V_{i}$ implies that the maps $\left(H_{t}\right)_{\#}: \pi_{1}\left(L_{0}, x_{0}\right) \rightarrow \pi_{1}\left(L_{t}, x_{t}\right)$ are injective for $t_{i} \leq t \leq t_{i+1}$, and Lemma 3.3 implies the isotropy group $H_{x_{i}} \subset$ $H_{x_{i} z_{i}(t)}$ for $t_{i} \leq t \leq t_{i+1}$. It is assumed that there exists an $\ell$-regular sequence $\left\{h_{1}, \ldots, h_{\ell}\right\} \subset H_{x_{i}}$ in the image of (13); hence $\left\{h_{1}, \ldots, h_{\ell}\right\}$ is an $\ell$-regular sequence in $H_{x_{i} z_{i+1}}$. Thus, $z_{0}(t) \in E^{\ell} \cap \mathcal{D}_{0}$ for $0 \leq t \leq t_{1}$. As $H_{t}$ is a foliated map, this implies $H_{t}\left(L_{0}\right) \subset E^{\ell}$ for $0 \leq t \leq t_{i+1}$. By the commutative diagram (11), it follows that there is an $\ell$-regular sequence in the image of

$$
h_{x_{i+1}} \circ\left(H_{t_{i+1}}\right)_{\#}: \pi_{1}\left(L_{0}, x_{0}\right) \rightarrow \pi_{1}\left(L_{x_{i+1}}, x_{i+1}\right) \rightarrow \mathbf{O}(q),
$$

as was to be shown.

The next result shows that homotopies in the exceptional set extend to open sets in the manifold. The subset $E$ and each stratum $E^{\ell}$ are assumed to have the relative topology. Let $\mathcal{F} \mid E^{\ell}$ denote the restricted foliation.

Theorem 5.4 (Extension). Let $V \subset E^{\ell}$ be a relatively open, saturated set in a compact manifold $M$. If $V$ is transversely categorical in $E^{\ell}$, then there exists a transversely categorical open saturated set $U \subset M$ with $V=U \cap E^{\ell}$.

Proof. Let $H: V \times[0,1] \rightarrow E^{\ell}$ be a foliated homotopy contracting $V$ to a leaf $L_{1} \subset E^{\ell}$.

The idea of the proof is to exhibit an open saturated set $W \subset M$ with $E^{\ell} \subset$ $W$, and a foliated homotopy $K: W \times[0,1] \rightarrow M$ such that $K_{0}$ is the identity, $K_{1}: W \rightarrow E^{\ell}$, and the restriction of $K_{t}$ to $E^{\ell}$ is the inclusion for all $t$. That is, we construct a foliated retract of $W$ to $E^{\ell}$. Then, given $V$ as above, $U=K_{1}^{-1}(V) \subset W$ is an open saturated set in $M$ such that $U \cap E^{\ell}=V$, and concatenation of the homotopies $H$ and $K$ provides a foliated homotopy of $U$ to $L_{1}$. The construction of $W$ and $K: W \times[0,1] \rightarrow M$ proceeds by induction, where we construct an increasing sequence of open sets $W_{1} \subset \cdots \subset W_{n}=W$ and foliated homotopies. We first need several preliminary results.

The set $E^{\ell}$ is compact, so we can choose points $\left\{x_{1}, \ldots, x_{n}\right\} \subset E^{\ell}$ and standard neighborhoods $\left(V_{i}, \phi_{i}, G_{i}, \alpha_{i}\right)$ so that $E^{\ell} \subset W=V_{1} \cup \cdots \cup V_{n}$. In the following, we again adopt the notational convention of replacing the subscript $x_{i}$ with the index $i$ when there is no chance of confusion.

For $r>0$ let $\mathbb{D}_{r}^{q} \subset \mathbb{R}^{q}$ denote the disk of radius $r$, and let $V_{i}^{r}=\phi_{i}\left(L_{0} \times_{G_{i}} \mathbb{D}_{r}^{q}\right)$. As $E^{\ell}$ is compact, there exists $s<1$ such that $\left\{V_{1}^{s}, \ldots, V_{n}^{s}\right\}$ also covers $E^{\ell}$. The homotopy $K$ will be defined as the concatenation of $n$ foliated homotopies, each with support in one of the $V_{i}$. These are constructed in turn using the following technical result:

Proposition 5.5. Let $x \in E^{\ell}$, and let $\left(V_{x}, \phi_{x}, G_{x}, \alpha_{x}\right)$ be a standard neighborhood. For any $0<s<s^{\prime}<1$ there exists a foliated homotopy $\Phi: M \times[0,1] \rightarrow M$ such that

(1) $\Phi_{0}=K \mid M \times\{0\}$ is the identity,

(2) $\Phi_{t}$ is the identity on $\left(M-V_{x}^{s^{\prime}}\right) \cup\left(V_{x} \cap E^{\ell}\right)$ for all $0 \leq t \leq 1$, and

(3) $\Phi_{1}=K \mid V_{x} \times\{1\}: V_{x} \rightarrow E^{\ell}$. 
Proof. We define $\Phi_{t}$ as the extension of a reparametrization of a flow $\phi_{t}$ of a vector field $X$ on $V_{x}$. We thank the referee for suggesting the following construction of the vector field. Recall that

$$
W_{x}^{\ell}=\bigcup_{\left[g_{1} \cdots g_{\ell}\right] \subset H_{x}} W\left(\left\langle g_{1}, \ldots, g_{\ell}\right\rangle\right) \subset \mathbb{D}^{q}
$$

is the $H_{x}$-invariant closed set defined by (8), so that by (10)

$$
E^{\ell} \cap V_{x}=\phi_{x}\left(L_{0} \times_{H_{x}} W_{x}^{\ell}\right) .
$$

For each $\ell$-regular sequence $\left\{g_{1} \cdots g_{\ell}\right\}$, define

$$
\rho_{\left[g_{1} \cdots g_{\ell}\right]}: \mathbb{D}^{q} \rightarrow[0,1], \rho_{\left[g_{1} \cdots g_{\ell}\right]}(y)=\operatorname{dist}\left(y, W\left(\left\langle g_{1}, \ldots, g_{\ell}\right\rangle\right)\right)^{2} .
$$

Then set

$$
\rho=\prod_{\left[g_{1} \cdots g_{\ell}\right] \subset H_{x}} \rho_{\left[g_{1} \cdots g_{\ell}\right]}: \mathbb{D}^{q} \rightarrow[0,1] .
$$

As the distance function dist is invariant under isometries, and the action of $H_{x}$ permutes the $\ell$-regular sequences, $\rho$ is an $H_{x}$-invariant function on $\mathbb{D}^{q}$.

Let $X=\nabla \rho$ be the gradient vector field of the function $\rho$, so that $X$ is also $H_{x}$-invariant. Note that $X$ vanishes exactly on $W_{x}^{\ell}$. Let $\varphi_{t}$ denote the flow of $X$.

Lemma 5.6. For all $0<r<1$, the flow $\varphi$ satisfies $\varphi_{t}\left(\mathbb{D}_{r}^{q}\right) \subset \mathbb{D}_{r}^{q}$ for all $0 \leq t<\infty$.

Proof. Let $r \partial / \partial r$ denote the gradient field of the radial distance squared function $r^{2}=\operatorname{dist}(y,\{0\})^{2}$. Then the inner product

$$
\langle r \partial / \partial r, X\rangle=\sum_{\left[g_{1} \cdots g_{\ell}\right] \subset H_{x}}\left\langle r \partial / \partial r, \nabla \rho_{\left[g_{1} \cdots g_{\ell}\right]}\right\rangle\left(\prod_{\left[h_{1} \cdots h_{\ell}\right] \neq\left[g_{1} \cdots g_{\ell}\right]} \rho_{\left[g_{1} \cdots g_{\ell}\right]}\right) .
$$

Each term $\left\langle r \partial / \partial r, \nabla \rho_{\left[g_{1} \cdots g_{\ell}\right]}\right\rangle \leq 0$ by the convexity of the distance squared function $r^{2}$ and the fact that $\nabla \rho_{\left[g_{1} \cdots g_{\ell}\right]}$ is the gradient flow of the distance squared function to a linear subspace. Each of the product terms in (15) is non-negative, so the sum of the products with the gradient functions is non-positive. Thus $\langle r \partial / \partial r, X\rangle \leq 0$, which implies that for any $y \in \mathbb{D}^{q}$, the function $\operatorname{dist}\left(\varphi_{t}(y),\{0\}\right)^{2}$ is decreasing as $t \rightarrow \infty$. Thus, $\varphi_{t}\left(\mathbb{D}_{r}^{q}\right) \subset \mathbb{D}_{r}^{q}$ for all $t \geq 0$.

Lemma 5.7. The fixed-point set of $\varphi_{t}$ is exactly $W_{x}^{\ell}$. For $y \notin W_{x}^{\ell}, \lim _{t \rightarrow \infty} \varphi_{t}(y)$ exists and is in $W_{x}^{\ell}$.

Proof. The first claim is obvious, as $X$ vanishes exactly on $W_{x}^{\ell}$.

For the second claim, note that the flow lines of the gradient field $X=\nabla \rho$ are orthogonal to the level curves $\rho=c^{2}$ for $c>0$. The orbits of a gradient-like vector field limit to points in the fixed-point set $\rho=0$ (cf. pages 322-325 in [35]), which is precisely $W_{x}^{\ell}$.

The flow $\varphi_{t}$ induces a flow $\tilde{\varphi}_{t}$ on the product space $L_{0} \times \mathbb{D}^{q}$ which preserves the product foliation on $L_{0} \times \mathbb{D}^{q}$. As the flow $\varphi$ is $H_{x}$-equivariant, the flow $\tilde{\varphi}_{t}$ descends to a flow $\phi_{t}$ of $L_{0} \times{ }_{H_{x}} \mathbb{D}^{q}$ which preserves the foliation $\mathcal{F} \mid V_{x}$. Let $\phi: V_{x} \times[0, \infty) \rightarrow V_{x}$ be given by $\phi(x, t)=\phi_{t}(x)$.

Recall that $s<s^{\prime}<1$ are given. Choose a smooth function $\lambda:[0,1] \rightarrow[0,1]$ such that $\lambda(r)=1$ for $0 \leq r \leq s$ and $\lambda(r)=0$ for $s^{\prime} \leq r \leq 1$. That is, $\lambda$ is a radial "cut-off" function on $\mathbb{D}^{q}$, and is $\mathbf{O}(q)$-invariant, hence $H_{x}$-invariant also. It thus descends to a radial distance function on $V_{x}$, again denoted by $\lambda$. 
Now define $\Phi: V_{x} \times[0,1] \rightarrow V_{x}$ by

$$
\Phi(x, t)=\phi(x, \arctan (t \cdot \lambda(x) \cdot \pi / 2)) .
$$

Note that for all $x \in V_{x}, \Phi(x, 0)=x$. For all $x \in\left(V_{x}-V_{x}^{s^{\prime}}\right) \cup\left(V_{x} \cap E^{\ell}\right), \Phi(x, t)=x$ for all $0 \leq t \leq 1$. For $x \notin E^{\ell}, \Phi(x, 1)=\lim _{t \rightarrow \infty} \phi_{t}(x) \in E^{\ell}$.

As each $\Phi_{t}$ is the identity on an open neighborhood of the boundary of $V_{x}$, we can extend it by the identity map on $M-V_{x}$ to a foliated homotopy $\Phi_{t}: M \rightarrow M$ which satisfies the claims of Proposition 5.5.

We now conclude the proof of Theorem 5.4. Recall that $s<1$ was chosen so that $\left\{V_{1}^{s}, \ldots, V_{n}^{s}\right\}$ covers $E^{\ell}$. Choose $s<s^{\prime}<1$. For each $i=1, \ldots, n$, let $\Phi^{i}: M \times[0,1] \rightarrow M$ be a foliated homotopy centered at $x_{i}$ as constructed in Proposition 5.5. Make a time-change in $\Phi^{i}$ by $t \rightarrow n \cdot t-(i-1)$, and denote the resulting reparametrized homotopy by $K^{i}$. Then $K^{i}$ is constant in $t$ for $t \leq(i-1) / n$ and for $t \geq i / n$, and implements $\Phi_{t}^{i}$ for $(i-1) / n \leq t \leq i / n$. Finally, we define

$$
K_{t}=K_{t}^{n} \circ \cdots \circ K_{t}^{1}: M \rightarrow M, 0 \leq t \leq 1 .
$$

Note that for $t=0, K_{0}$ is the identity map. Moreover, $K_{t}$ is the identify on $E^{\ell}$ for $0 \leq t \leq 1$.

Lemma 5.8. There exists an open neighborhood $E^{\ell} \subset W$ such that $K_{1}: W \rightarrow E^{\ell}$.

Proof. The proof is by induction. We define an increasing sequence of open sets $W_{1} \subset \cdots \subset W_{n}$ such that for $1 \leq k \leq n$,

(1) $E^{\ell} \cap\left(V_{1}^{s} \cup \cdots \cup V_{k}^{s}\right) \subset W_{k} \subset V_{1}^{s} \cup \cdots \cup V_{k}^{s}$,

(2) $K_{k / n}^{k} \circ \cdots \circ K_{k / n}^{1}\left(W_{k}\right) \subset E^{\ell}$, and

(3) $E^{\ell} \subset W=W_{n}$.

The homotopy $K_{t}^{1}: M \rightarrow M$ satisfies $K_{1 / n}^{1}\left(V_{1}^{s}\right) \subset E^{\ell}$. The partial composition $K_{t}^{n} \circ \cdots \circ K_{t}^{2}: M \rightarrow M$ is constant on $E^{\ell}$, and the identity for $0 \leq t \leq 1 / n$, so $K_{t}$ retracts $V_{1}^{s}$ to $E^{\ell}$. Set $W_{1}=V_{1}^{s}$.

Let $1 \leq k<n$ and assume that $W_{k}$ has been defined satisfying conditions (1) and (2) above. Define

$$
W_{k+1}=W_{k} \cup\left(V_{k+1}^{s} \cap\left(K_{k / n}^{k} \circ \cdots \circ K_{k / n}^{1}\right)^{-1}\left(V_{k+1}^{s}\right)\right) .
$$

The restriction $K_{k / n}^{k} \circ \cdots \circ K_{k / n}^{1}: E^{\ell} \rightarrow E^{\ell}$ is the identity, so

$$
\left(E^{\ell} \cap V_{k+1}^{s}\right) \subset\left(K_{k / n}^{k} \circ \cdots \circ K_{k / n}^{1}\right)^{-1}\left(V_{k+1}^{s}\right),
$$

whence $E^{\ell} \cap\left(V_{1}^{s} \cup \cdots \cup V_{k+1}^{s}\right) \subset W_{k+1}$. By the inductive hypotheses, $K_{k / n}^{k} \circ \cdots \circ$ $K_{k / n}^{1}\left(W_{k}\right) \subset E^{\ell}$, so

$$
K_{k / n}^{k} \circ \cdots \circ K_{k / n}^{1}\left(V_{k+1}^{s} \cap\left(K_{k / n}^{k} \circ \cdots \circ K_{k / n}^{1}\right)^{-1}\left(V_{k+1}^{s}\right)\right) \subset V_{k+1}^{s} .
$$

It follows that

$$
K_{(k+1) / n}^{k+1} \circ \cdots \circ K_{(k+1) / n}^{1}\left(W_{k+1}\right) \subset E^{\ell},
$$

so the inductive step is completed.

To complete the proof of Theorem [5.4 we concatenate the given homotopy $H: V \times[0,1] \rightarrow E^{\ell}$ with the homotopy $K: U \times[0,1] \rightarrow M$, where $U=K_{1}^{-1}(V) \cap$ $W$. 


\section{Estimates OF THE TRANSVERSE CATEGORY}

In this section, we formulate and prove estimates for the transverse category of compact Hausdorff foliations. In the following section we give examples to show that these estimates are optimal.

Theorem 6.1. Let $\mathcal{F}$ be a compact Hausdorff foliation of a compact manifold $M$ with non-empty exceptional set E. Then

$$
\max \left\{\operatorname{cat}(M / \mathcal{F}), \operatorname{cat}_{\phi}(E)\right\} \leq \operatorname{cat}_{\pitchfork}(M) \leq \operatorname{cat}_{\pitchfork}(E)+q .
$$

Proof. The $\mathcal{F}$-saturation of an open subset of $M$ is open, so $\pi: M \rightarrow M / \mathcal{F}$ is an open map. Suppose that $H: U \times[0,1] \rightarrow M$ is a foliated homotopy contracting $U$ to a leaf $L$. Then $U / \mathcal{F}$ is open in $M / \mathcal{F}$ and $\pi \circ H: U \times[0,1] \rightarrow M / \mathcal{F}$ induces a contraction of $U / \mathcal{F}$ to the point $b=\pi(L)$. Thus, if $\left\{U_{1}, \ldots, U_{n}\right\}$ is a covering of $M$ by transversely categorical saturated open sets, then $\left\{U_{1} / \mathcal{F}, \ldots, U_{n} / \mathcal{F}\right\}$ is a categorical covering of $M / \mathcal{F}$, so $\operatorname{cat}(M / \mathcal{F}) \leq \operatorname{cat}_{巾}(M)$.

Next, suppose $\left\{U_{1}, \ldots, U_{n}\right\}$ is a covering of $M$ by transversely categorical saturated open sets. For each $U_{k}$ let $V_{k}=U_{k} \cap E$ be the relatively open (possibly empty) subset of $E$. Then by Theorem 5.3 each $V_{k}$ is transversely categorical, and hence $\operatorname{cat}_{\pitchfork}(E) \leq \operatorname{cat}_{\pitchfork}(M)$.

Let $\left\{V_{1}, \ldots, V_{k}\right\}$ be a covering of $E$ by transversely categorical open saturated sets for the relative topology on $E$ and restricted foliation $\mathcal{F} \mid E$, where $k=\operatorname{cat}_{\phi}(E)$. By Theorem 5.4 we can extend each $V_{i}$ to a transversely categorical open saturated set $U_{i}$ for $\mathcal{F}$, and then $\left\{U_{1}, \ldots, U_{k}\right\}$ is a covering of $E$ by transversely categorical saturated open sets in $M$.

The good set $G=M-E$ is a fibration over the quotient space $G / \mathcal{F}$, which is an open manifold of dimension $q$. Thus $G / \mathcal{F}$ admits a retract to a $(q-1)$ dimensional $\mathrm{CW}$ complex, and so the category of $G / \mathcal{F}$ is at most $q$. Given a categorical covering of $G / \mathcal{F}$ by open sets $\left\{W_{1}, \ldots, W_{q}\right\}$, for each $1 \leq i \leq q$ set $U_{k+i}=\pi^{-1}\left(W_{i}\right)$, which is a transversely contractible open saturated set in $M$. Then $\left\{U_{1}, \ldots, U_{k}, U_{k+1}, \ldots, U_{k+q}\right\}$ is a transversely categorical covering of $M$, so $\operatorname{cat}_{\pitchfork}(M) \leq \operatorname{cat}_{\pitchfork}(E)+q$

When $E$ consists of a finite collection of exceptional leaves, $\operatorname{cat}_{\pitchfork}(E)$ equals the cardinal $N_{E}$ of the quotient space $E / \mathcal{F}$. In this special case we obtain the estimate:

Corollary 6.2. Let $\mathcal{F}$ be a compact Hausdorff foliation of a compact manifold $M$, and suppose $E$ consists of a finite number $N_{E}$ of exceptional leaves. Then

$$
\max \left\{\operatorname{cat}(M / \mathcal{F}), N_{E}\right\} \leq \operatorname{cat}_{\pitchfork}(M) \leq N_{E}+q
$$

Note that the proof of the estimate $\operatorname{cat}_{\phi}(E) \leq \operatorname{cat}_{\phi}(M)$ in Theorem 6.1 extends to $\operatorname{show}_{\operatorname{cat}}\left(E^{\ell}\right) \leq \operatorname{cat}_{\phi}\left(E^{\ell-1}\right)$ for all $1 \leq \ell \leq k$. Hence we have the estimates

Corollary 6.3. Let $\mathcal{F}$ be a compact Hausdorff foliation of a compact manifold $M$. Then

$$
\operatorname{cat}_{\phi}\left(E^{k}\right) \leq \operatorname{cat}_{\pitchfork}\left(E^{k-1}\right) \leq \cdots \leq \operatorname{cat}_{\pitchfork}(E) \leq \operatorname{cat}_{\pitchfork}(M) .
$$

We say that a compact Hausdorff foliation $(M, \mathcal{F})$ is $\operatorname{good}$ if there is a Galois covering $p: \tilde{M} \rightarrow M$ with finite covering group $\Delta$, such that the lifted foliation $\tilde{\mathcal{F}}$ defines a fibration $\tilde{M} \rightarrow \tilde{B}$ over the base manifold $B$. We say that $(p: \tilde{M} \rightarrow$ $M, \tilde{\mathcal{F}}, \Delta)$ is a good covering of $\mathcal{F}$. 
If $\bar{p}: \bar{M} \rightarrow \tilde{M}$ is a finite Galois covering of a good covering $p: \tilde{M} \rightarrow M$, then the composition $\hat{p}=p \circ \bar{p}: \bar{M} \rightarrow M$ is again a finite Galois covering whose Galois group is an extension $\hat{\Delta}$ of $\Delta$ by $\bar{\Delta}$, and for the lifted foliation $\overline{\mathcal{F}}$ on $\bar{M}$ the data $(\hat{p}: \bar{M} \rightarrow M, \overline{\mathcal{F}}, \hat{\Delta})$ is again a good covering of $\mathcal{F}$.

Consider a finite Galois covering $\tilde{p}: \tilde{M} \rightarrow M$, and let $\tilde{\mathcal{F}}$ be a lifted foliation whose leaves cover those of $\mathcal{F}$. Since all leaves of $\mathcal{F}$ are compact and the covering is finite, the leaves of $\tilde{\mathcal{F}}$ are again compact. Thus, $\tilde{\mathcal{F}}$ defines a fibration exactly when every leaf of $\tilde{\mathcal{F}}$ has no holonomy. Note that a leaf with holonomy cannot be simply connected. Thus, if $(M, \mathcal{F})$ is not good, then for every finite Galois covering of $M$, there is a leaf of the lifted foliation whose fundamental group is non-trivial.

Lee and Raymond [27] say that a Seifert fibre space $(M, \mathcal{F})$ is injective if the fundamental group of each leaf injects into that of $M$. Each leaf of $\mathcal{F}$ is then essential is the sense of 3-manifolds. The following is a nice exercise.

Proposition 6.4. Let $\mathcal{F}$ be a compact Hausdorff foliation of a compact manifold $M$. Suppose that $(M, \mathcal{F})$ is injective, and the fundamental group $\pi_{1}(M)$ is residually finite. Then $(M, \mathcal{F})$ is good.

Lee and Raymond also give many examples of injective Seifert fiber spaces on manifolds whose fundamental groups are residually finite. Hence these are all good compact Hausdorff foliations.

For a good compact Hausdorff foliation, we can improve the estimates of Theorem 6.1 .

Let $(p: \tilde{M} \rightarrow M, \tilde{\mathcal{F}}, \Delta)$ be a good covering of $\mathcal{F}$. The leaf space $\tilde{M} / \tilde{\mathcal{F}}$ is naturally identified with $\tilde{B}$, and we have the commutative diagram

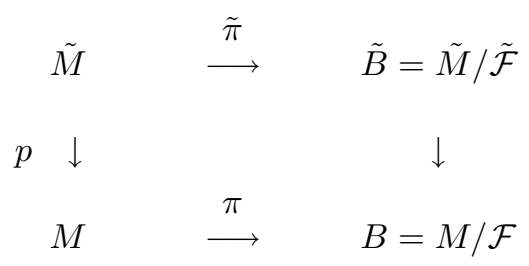

The leaves of $\tilde{\mathcal{F}}$ are compact without holonomy, so the restriction $p: \tilde{L} \rightarrow L$ to a leaf $\tilde{L}$ of $\tilde{\mathcal{F}}$ is the holonomy covering of $L$. Thus, the holonomy group of $L$ is isomorphic to the stabilizer subgroup $\Delta_{L} \subset \Delta$ of $\tilde{L}$.

The covering action of $\Delta$ on $\tilde{M} \rightarrow M$ maps leaves to leaves, hence induces an action on $\tilde{B}$. The set of points in $\tilde{B}$ with non-trivial isotropy group corresponds to the set of leaves of $\mathcal{F}$ in $M$ with holonomy. Thus, $\tilde{B} \rightarrow M / \mathcal{F}=B$ is an "orbifold covering" in the sense of Thurston [40. For example, a 3-manifold $M$ foliated by circles is a compact Hausdorff foliation [11, hence is a Seifert fibration, and the leaf space $B$ is the corresponding 2 -dimensional orbifold. If $\mathcal{F}$ is good, then $B$ is a good orbifold with covering $\tilde{B}$.

For a compact Hausdorff foliation of codimension greater than 2, the leaf space $B$ is said to be a generalized orbifold, in analogy with the 2-dimensional case. However, a generalized orbifold need not be a manifold, and while the quotient $G / \Delta$ is a manifold, the action of $\Delta$ in a normal neighborhood of the exceptional set $E$ can be exotic, and the quotient space $B$ need not be manifold-like near $E / \Delta$. 
Varadarajan's Theorem states that the category of a covering is always less than or equal to the category of the base [42]. We show that the generalization of this holds in the foliated category for a good compact Hausdorff foliation.

Theorem 6.5. Let $(p: \tilde{M} \rightarrow M, \tilde{\mathcal{F}}, \Delta)$ be a good covering. Then

$$
\operatorname{cat}_{\pitchfork}(\tilde{M})=\operatorname{cat}(\tilde{B}) \leq \operatorname{cat}_{\phi}(M) .
$$

Proof. Let $\left\{U_{1}, \ldots, U_{k}\right\}$ be a categorical covering of $M$. For each $1 \leq i \leq k$, the lift $\tilde{U}_{i}$ is a finite union of disjoint connected open subsets of $\tilde{M}$ such that $p$ restricted to each is a covering.

Let $H_{t}^{i}: U_{i} \times[0,1] \rightarrow M$ be a foliated homotopy such that the image of $H_{1}^{i}$ is contained in a leaf of $\mathcal{F}$. Then by the covering homotopy property of $p, H^{i}$ lifts to each connected component of $\tilde{U}_{i}$. This yields a foliated homotopy $\tilde{H}_{t}^{i}$ for $\tilde{\mathcal{F}}$ which maps each connected component of $\tilde{U}_{i}$ to a leaf of $\tilde{\mathcal{F}}$. As $\tilde{\mathcal{F}}$ is a fibration, all leaves of $\tilde{\mathcal{F}}$ are isotopic, so we can concatenate the homotopy $\tilde{H}_{t}^{i}$ with a homotopy of the leaves in the image of $\tilde{H}_{1}^{i}$ into one leaf of $\tilde{\mathcal{F}}$. Hence $\tilde{U}_{i}$ is transversely categorical for $\tilde{\mathcal{F}}$, and thus $\left\{\tilde{U}_{1}, \ldots, \tilde{U}_{k}\right\}$ is a categorical covering of $\tilde{M}$, so $\operatorname{cat}_{巾}(\tilde{M}) \leq k=$ $\operatorname{cat}_{\pitchfork}(M)$.

In order to show that (19) is an equality, it would suffice to show that, given a transversely categorical open saturated set $\tilde{U} \subset \tilde{M}$, its projection $U \subset M$ is transversely categorical for $\mathcal{F}$. For example, if the translates of the connected components of $\tilde{U}$ by the covering transformations are disjoint, then the restriction of a foliated homotopy to one of the connected components descends to a foliated homotopy of the quotient open set $U$. Otherwise, $U$ need not be contractible in $M$.

Another obstacle to $U$ being transversely categorical can arise from the singular set $E$ of $\mathcal{F}$. The singular set $\tilde{E}$ of $\tilde{\mathcal{F}}$ is empty by definition, so a foliated homotopy $H: \tilde{U} \times[0,1] \rightarrow \tilde{M}$ does not necessarily preserve the leaves of $\tilde{\mathcal{F}}$ covering those of the exceptional set $E \subset M$. However, a foliated homotopy of $U \subset M$ must preserve $E \cap U$, which imposes an additional restraint.

For a good compact Hausdorff foliation $(M, \mathcal{F})$ with good covering $(p: \tilde{M} \rightarrow$ $M, \tilde{\mathcal{F}}, \Delta)$ one can also define the $\Delta$-equivariant category $\operatorname{cat}_{\Delta}(\tilde{B})$. This category depends on the basic filtration of the group action [7, which is analogous to the Epstein filtration studied in this paper. Thus, the following conjecture is natural, and supported by examples.

Conjecture 6.6. Let $(M, \mathcal{F})$ be a good compact Hausdorff foliation and $(p: \tilde{M} \rightarrow$ $M, \tilde{\mathcal{F}}, \Delta)$ a good covering of $\mathcal{F}$. Then $\operatorname{cat}_{\phi}(M)=\operatorname{cat}_{\Delta}(\tilde{B})$.

\section{EXAMPLES}

In this section, we consider selected examples of compact Hausdorff foliations with non-trivial exceptional set $E$. These examples illustrate each of the lower bounds in the estimate

$$
\max \left\{\operatorname{cat}(B), \operatorname{cat}(\tilde{B}), \operatorname{cat}_{\phi}(E)\right\} \leq \operatorname{cat}_{\phi}(M) \leq \operatorname{cat}_{\phi}(E)+q
$$

derived from Theorems 6.1 and 6.5. (Set $\operatorname{cat}(\tilde{B})=0$ if $\tilde{B}$ does not exist.)

Example 7.4 is of special interest, as it provides examples where cat ${ }_{\phi}(M)$ can be made arbitrarily large, even though $\operatorname{cat}(B)=2$ remains constant.

The upper bound cat ch $(E)+q$ on the category is illustrated in Example 7.5 . 

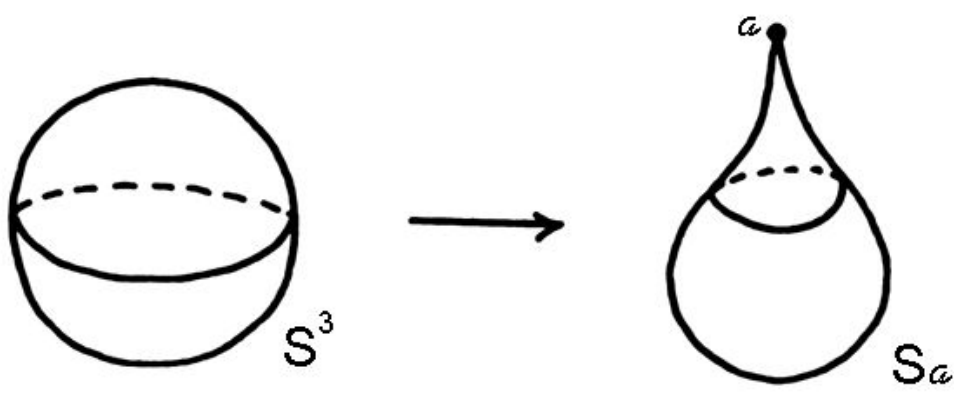

FiguRe 2.

Example 7.1. A bad Seifert foliation on $S^{3}$.

In this example, $1=\operatorname{cat}_{\phi}(E)<\operatorname{cat}(B)=2=\operatorname{cat}_{\pitchfork}\left(S^{3}\right)<\operatorname{cat}_{\pitchfork}(E)+2=3$.

We define a foliation $\mathcal{F}_{a, b}$ of the 3 -sphere as the orbits of a locally free action of $\mathbb{R}$. Let $S^{3}=\{[z, w] \mid z \bar{z}+w \bar{w}=1\}$. For integers $a, b$ with $(a, b)=1$, set $t \cdot[z, w]=\left[e^{2 \pi i a t} \cdot z, e^{2 \pi i b t} \cdot w\right]$. If $z \cdot w \neq 0$, then the orbit of $[z, w]$ is a closed circle of length $2 \pi \sqrt{(a\|z\|)^{2}+(b\|w\|)^{2}}$, while the orbits of $[z, 0]$ and $[0, w]$ are closed circles of length $2 \pi$. Thus, the exceptional set of $\mathcal{F}_{a, b}$ consists of the orbits of $[1,0]$ and $[0,1]$. The holonomy group $H_{[1,0]} \subset \mathbf{O}(\mathbf{2})$ is isomorphic to $\mathbb{Z} / a \mathbb{Z}$, and $H_{[0,1]} \subset \mathbf{O}(\mathbf{2})$ is isomorphic to $\mathbb{Z} / b \mathbb{Z}$.

If $a=b=1$, then $\mathcal{F}_{1,1}$ is just the Hopf fibration of $S^{3}$, and the quotient space $B=S^{2}$. When $a>1$ and $b=1$, the quotient space $B$ is a singular orbifold $S a$ homeomorphic to $S^{2}$, pictured in Figure 2. The quotient $S a$ is a bad orbifold, so $\mathcal{F}_{a, 1}$ cannot be a good compact Hausdorff foliation. We have $\operatorname{cat}(B)=\operatorname{cat}(S a)=2$, $\operatorname{cat}_{\pitchfork}(E)=N_{E}=1$ and $\operatorname{cat}_{巾}\left(S^{3}\right)=2$.

Example 7.2. A good Seifert foliation on a 3-manifold.

In this example, $\operatorname{cat}_{\pitchfork}(M)=\operatorname{cat}(B)$ again, but $B$ is a good orbifold that admits a covering orbifold which is $\tilde{B}=\Sigma_{2}$.

Let $\Sigma_{2}$ be the genus 2 surface, and let $G \cong \mathbb{Z} / 2 \mathbb{Z}$ be generated by the involution $\alpha$ of $\Sigma_{2}$ obtained by rotating $\Sigma_{2} 180$ degrees around the central axis, as illustrated in Figure 3. The generic leaf $L$ is $\mathbb{S}^{1}$ with $\alpha$ acting via rotation by 180 degrees. Define $\tilde{M}=\Sigma_{2} \times \mathbb{S}^{1}$ foliated by circles, so $\tilde{B}=\Sigma_{2}$. The quotient $M=\Sigma_{2} \times_{G} \mathbb{S}^{1}$ has exceptional set $E$ consisting of 2 circles which project to the 2 cusp points in $B=\Sigma_{2} / G$ as shown on the right in Figure 3 .

Then $\operatorname{cat}(\tilde{B})=\operatorname{cat}(B)=3$ and $\operatorname{cat}_{\pitchfork}(E)=2$. A transversely categorical cover for $M$ is given by the three open sets $\left\{U_{1}, U_{2}, U_{3}\right\}$ whose projections to $B$ are indicated in Figure 4. Thus $\operatorname{cat}_{巾}(M)=3$.

Example 7.3. A good Seifert foliation on a 4-manifold.

In this example, $\operatorname{cat}_{\phi}(M)=\operatorname{cat}_{\phi}(E)$. More precisely,

$$
\operatorname{cat}(B)=2<\operatorname{cat}(\tilde{B})=3<\operatorname{cat}_{\pitchfork}(E)=4=\operatorname{cat}_{\pitchfork}(M)<\operatorname{cat}_{\pitchfork}(E)+2=6 .
$$

Let $\Sigma_{2}$ be a genus 2 closed surface as pictured on the left in Figure 5, and $G \cong \mathbb{Z} / 2 \mathbb{Z} \times \mathbb{Z} / 2 \mathbb{Z}$ the group of order 4 generated by two involutions $\{\alpha, \beta\}$ of $\Sigma_{2}$. 


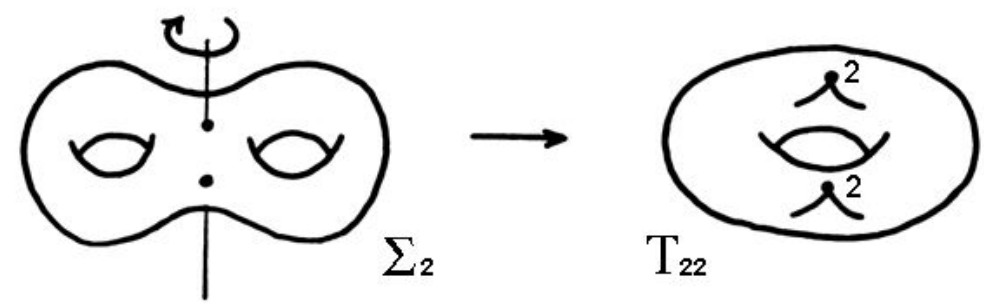

FiguRE 3.

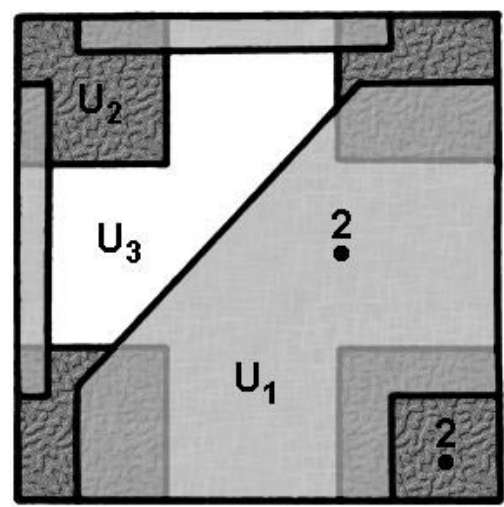

FIGURE 4.

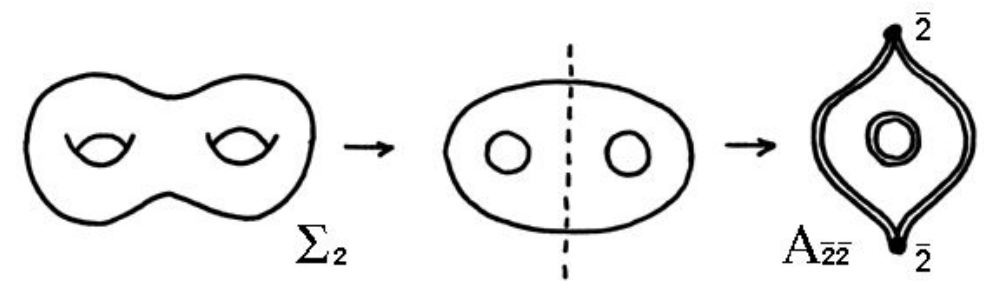

FIGURE 5

The action of $\alpha$ is the front-back involution of $\Sigma_{2}$. In the middle of Figure 5 is the quotient $\Sigma_{2} / \alpha$. The action of $\beta$ is the left-right involution $\Sigma_{2}$. Shown on the right is the quotient $\Sigma_{2} / G$.

We next need a free action of $G$ on the generic leaf $L$. Unfortunately, $\mathbb{Z} / 2 \mathbb{Z} \times \mathbb{Z} / 2 \mathbb{Z}$ cannot act freely on $\mathbb{S}^{1}$, as rotation by 180 degrees is the unique free action of $\mathbb{Z} / 2 \mathbb{Z}$ on $\mathbb{S}^{1}$. Instead, we let $\alpha$ act on $\mathbb{T}^{2}=\mathbb{S}^{1} \times \mathbb{S}^{1}$ by a 180 degree rotation in the first factor, and let $\beta$ act by a 180 degree rotation in the second factor.

Define $\tilde{M}=\Sigma_{2} \times \mathbb{T}^{2}$ foliated by the $\mathbb{T}^{2}$ factor, with $\tilde{B}=\Sigma_{2}$. The quotient $M=\Sigma_{2} \times_{G} \mathbb{T}^{2}$ has exceptional set $E$ consisting of components which project to the two boundary circles at the right in the picture of $B=\Sigma_{2} / G$.

Note that $\operatorname{cat}(B)=2, \operatorname{cat}(\tilde{B})=3$ and $\operatorname{cat}_{\phi}(E)=4$. A transversely categorical cover for $E$ extends to a transversely categorical cover for $M$; hence $\operatorname{cat}_{巾}(M)=4$. 


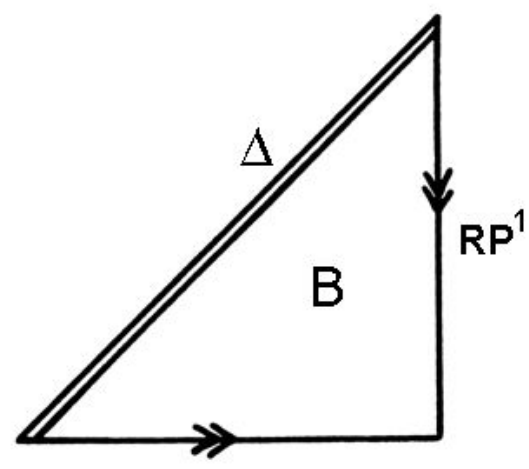

FiguRE 6.

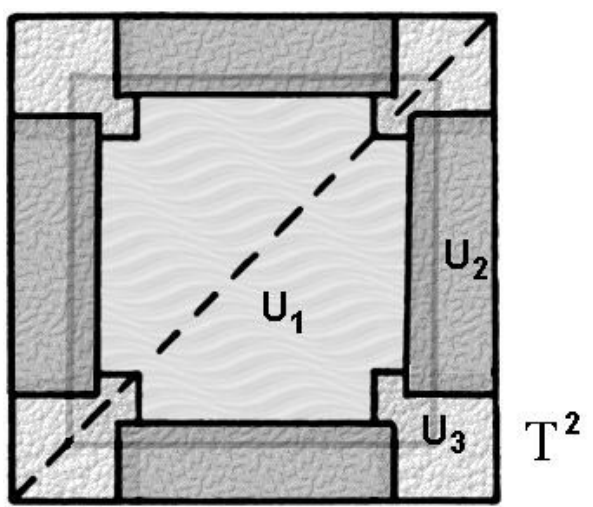

FiguRE 7.

Example 7.4. Equality of covering category.

In this example, $\operatorname{cat}_{\pitchfork}(M)=\operatorname{cat}(\tilde{B})$, while

$$
2=\operatorname{cat}(B)=\operatorname{cat}_{\phi}(E)<\operatorname{cat}(\tilde{B})=3=\operatorname{cat}_{\phi}(M)<\operatorname{cat}_{\phi}(E)+2=4
$$

Let $\tilde{B}=\mathbb{T}^{2}$ be the 2-torus, and let $G=\mathbb{Z} / 2 \mathbb{Z}$ act by the "diagonal" reflection on $\mathbb{T}^{2}$ about the diagonal $\Delta$, with fixed-set a circle in $\mathbb{T}^{2}$. The quotient space $B=\mathbb{T}^{2} / G$ admits a homotopy retraction onto an embedded $\mathbb{R} \mathbb{P}^{1}$, that is, a circle; hence it has category 2. See Figure 6.

The generic leaf $L$ is $\mathbb{S}^{1}$ with $\alpha$ acting via rotation by 180 degrees. Define $\tilde{M}=\mathbb{T}^{2} \times \mathbb{S}^{1}$ foliated by circles. The quotient $M=\mathbb{T}^{2} \times{ }_{G} \mathbb{S}^{1}$ has exceptional set $E$ consisting of a torus which projects to the diagonal quotient - a circle - in $B$. Thus, $\operatorname{cat}_{巾}(E)=2$.

Since $\operatorname{cat}_{巾}(M) \geq \operatorname{cat}(\tilde{B})=\mathbb{T}^{2}=3$, it suffices to exhibit a transversely categorical open covering of $M$ with 3 sets to complete the example.

We exhibit in Figure 7 a $G$-equivariant categorical covering of $\tilde{B}=\mathbb{T}^{2}$ by 3 open sets $\left\{U_{1}, U_{2}, U_{3}\right\}$. Then the saturated open subsets $\left\{U_{1} \times{ }_{G} \mathbb{S}^{1}, U_{2} \times{ }_{G} \mathbb{S}^{1}, U_{3} \times{ }_{G} \mathbb{S}^{1}\right\}$ of $M$ are transversely categorical. 


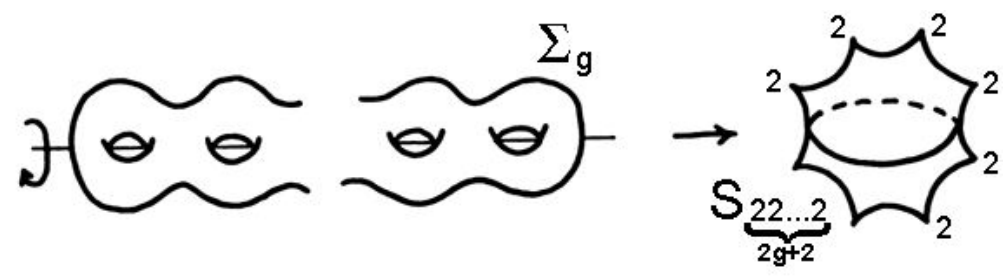

FIGURE 8.

Example 7.5. A sharp upper bound estimate.

In this example, we construct foliations of codimension 2 with $\operatorname{cat}_{\text {巾 }}(M)=$ $\operatorname{cat}_{\phi}(E)+2$. This realizes the upper bound in the estimate (16).

Let $\tilde{B}=\mathbb{R P}^{2}$ be the real projective space of dimension 2 . The group $G=$ $\mathbb{Z} / 3 \mathbb{Z}$ (though $G=\mathbb{Z} / p \mathbb{Z}$ for $p$ any odd prime will also work). Define the action $\phi: \mathbb{Z} / 3 \mathbb{Z} \times \mathbb{R} \mathbb{P}^{2} \rightarrow \mathbb{R P}^{2}$ to be the quotient of a rotation by $2 \pi / 3$ on the covering 2 -sphere $\mathbb{S}^{2}$. The rotation has 2 fixed points on $\mathbb{S}^{2}$, denoted by $\{ \pm a\}$. As $G$ has odd order, the quotient action on $\mathbb{R P}^{2}$ has a unique fixed-point, denoted by $[a]$.

Let $\tilde{M}=\mathbb{S}^{1} \times \mathbb{R P}^{2}$. Let $G$ act on the first factor $\mathbb{S}^{1}$ by a rotation, and act on the second factor $\mathbb{R P}^{2}$ as above. Then the quotient $M=\mathbb{S}^{1} \times_{G} \mathbb{R P}^{2}$ has a codimension 2 foliation $\mathcal{F}$, whose leaf space $B$ is identified with $\mathbb{R}^{2}$. Hence, $3=\operatorname{cat}(B)$.

On the other hand, $E$ consists of a single circle corresponding to the point $[a]$; hence $\operatorname{cat}_{\pitchfork}(E)=1$. Thus,

$$
3=\operatorname{cat}(B) \leq \operatorname{cat}_{巾}(M) \leq \operatorname{cat}_{巾}(E)+2=3 .
$$

This example can be generalized to higher codimensions.

Example 7.6. Arbitrarily large category.

In this example we show that cat $\cos _{\phi}(M)$ can be arbitrarily large while cat $(B)=2$. Let $\Sigma_{g}$ be the genus-g surface, and let $G \cong \mathbb{Z} / 2 \mathbb{Z}$ be generated by the involution $\alpha$ of $\Sigma_{g}$ obtained by rotating $\Sigma_{g} 180$ degrees around the horizontal axis, as illustrated in Figure 8. The generic leaf $L$ is $\mathbb{S}^{1}$ with $\alpha$ acting via rotation by 180 degrees. Define $\tilde{M}=\Sigma_{g} \times \mathbb{S}^{1}$ foliated by circles, so $\tilde{B}=\Sigma_{g}$. The quotient $M=\Sigma_{g} \times{ }_{G} \mathbb{S}^{1}$ has exceptional set $E$ consisting of $2 g+2$ circles which project to the the $2 g+2$ cusp points in $B=\Sigma_{g} / G$ as pictured on the right in Figure 8.

Then $\operatorname{cat}(B)=2, \operatorname{cat}(\tilde{B})=3$ and $\operatorname{cat}_{\pitchfork}(E)=2 g+2$. A transversely categorical cover for $E$ with $2 g+2$ open sets can be chosen to cover all of $M$; hence cat cat $(M)=$ $2 g+2$.

\section{REFERENCES}

[1] F. Bonahon and L. Siebenmann. The classification of Seifert fibred 3-orbifolds. In Lowdimensional topology (Chelwood Gate, 1982), Lond. Math. Soc. Lect. Note Ser., vol. 95, Cambridge Univ. Press, Cambridge (1985), pp. 19-85. MR 87k:57012

[2] G.E. Bredon. Introduction to compact transformation groups. Academic Press, New York, 1972. MR 54:1265]

[3] C. Camacho and A. Neto. Geometric Theory of Foliations. Progress in Math. Birkhausser, Boston, Basel and Stuttgart, 1985. MR 87a:57029

[4] A. Candel and L. Conlon. Foliations 1. Amer. Math. Soc., Providence, RI, 2000. MR 2002f: 57058 
[5] H. Colman. Categoría LS en foliaciones. Publicaciones del Departamento de Topología y Geometría, N 93, 1998, Universidade de Santiago de Compostele.

[6] H. Colman. LS-categories for foliated manifolds. Foliations: Geometry and Dynamics (Warsaw, 2000) World Scientific Publishing, River Edge, NJ. 2002:17-28. MR 2002m:55007

[7] H. Colman. Equivariant LS-category for finite group actions. In Proceedings of the AMS Conference: Lusternik-Schnirelmann Category in the New Millennium, July 2001, ed. O. Cornea et. al, Contemp. Math., vol. 316, Amer. Math. Soc., Providence, R.I., 2002, 35-40.

[8] H. Colman. Transverse category of Riemannian foliations. submitted, 2003.

[9] H. Colman and E. Macias. Transverse LS-category of foliated manifolds. Topology, Vol.40, 419-430, 2000. MR 2002c:55010

[10] R. Edwards, K. Millett, and D. Sullivan. Foliations with all leaves compact. Topology, 16:1332, 1977. MR 55:11268

[11] D. B. A. Epstein. Periodic flows on 3-manifolds. Annals of Math., 95:68-82, 1972. MR 44:5981

[12] D. B. A. Epstein. Foliations with all leaves compact. Ann. Inst. Fourier (Grenoble)., 26:265282, 1976. MR 54:8664

[13] D. B. A. Epstein and E. Vogt A counter-example to the Periodic Orbit Conjecture in codimension 3. Annals of Math., 108:539-552, 1978. MR 80c:57014

[14] E. Fadell. The equivariant Lusternik-Schnirelmann method for invariant functionals and relative cohomological index theories. In Méthodes Topologiques en Analyse Non-Lineaire, ed. A. Granas, Montreal, 1985, 41-70. MR 86f:58005

[15] E. Fadell and S. Husseini. Relative cohomological index theories. Adv. Math, 64:1-31, 1987. MR 88f:55006

[16] J. Feldman and C.C Moore. Ergodic equivalence relations, cohomology and von Neumann algebras, I. Trans. Amer. Math. Soc., 234:289-324, 1977. MR 58:28261a

[17] A. Haefliger. Groupoïdes d'holonomie et classifiants. Astérisque, 116:70-97, Société Mathématique de France 1984. MR 86c:57026a

[18] A. Haefliger. Complexes of groups and orbihedra. Group theory from a geometrical viewpoint (Trieste, 1990), World Sci. Publishing, River Edge, NJ, 1991, 504-540. MR 93m:20048

[19] H. Holmann. Seifertsche Faserräume. Math. Ann., 157:138-166, 1964. MR 30:587

[20] S. Hurder. Category and compact leaves. submitted, 2003.

[21] S. Hurder and A. Katok. Ergodic theory and Weil measures for foliations. Annals of Math., pages 221-275, 1987. MR 89d:57042

[22] S. Hurder and P. Walczak. Compact foliations with finite transverse category. preprint, 2002.

[23] I.M. James. On category, in the sense of Lusternik-Schnirelmann. Topology 17:331-348, 1978. MR 80i:55001

[24] I.M. James. Lusternik-Schnirelmann Category. Handbook of Algebraic Topology, (ed. I. M. James), Elsevier Science, Amsterdam, 1995, 1293-1310. MR 97a:55003

[25] I.M. James and J. Morris. Fibrewise category. Proceedings of the Royal Society of Edinburgh, 119A:177-190, 1991. MR 92g:55005

[26] M. Jankins and W.D. Neumann. Lectures on Seifert Manifolds. Brandeis Lecture Notes No. 2, March 1983. MR 85j:57015

[27] K.B. Lee and F. Raymond. The role of Seifert fiber spaces in transformation groups. In Group actions on manifolds (Boulder, Colo., 1983), Contemp. Math Vol. 36, pages 367-425, 1985. Amer. Math. Soc., Providence, RI. MR 86j:57015

[28] L. Lusternik and L. Schnirelmann. Méthodes topologiques dans les Problèmes Variationnels. Hermann, Paris, 1934.

[29] W. Marzantowicz. A G-Lusternik-Schnirelmann category of space with an action of a compact Lie group. Topology, 28:403-412, 1989. MR 91c:55002

[30] K. Millett. Compact foliations. In Foliations: Dijon 1974, Lect. Notes in Math. volume 484, pages 277-287,1975. Springer-Verlag, New York and Berlin. MR 52:11944

[31] C. C. Moore and C. Schochet. Analysis on Foliated Spaces. Math. Sci. Res. Inst. Publ., 9, Springer-Verlag, 1988. MR 89h:58184

[32] D. Montgomery. Compact groups of transformations. In Differential Analysis, Bombay Colloq., 1964, pages 43-56, 1964. Oxford Univ. Press, London. MR 31:6238

[33] R. Palais. The Classification of G-spaces. Memoirs of the Amer. Math Soc., no. 36. Amer. Math. Soc., Providence, RI 1960. MR 31:1664 
[34] G. Reeb. Sur certaines propiétés topologiques des variétés feuilletés. Act. Sci. et Ind. 1183:91154, 1952, Hermann, Paris. MR 14:1113a

[35] C. Robinson. Dynamical Systems. Stability, Symbolic Dynamics, and Chaos. CRC Press, 1995. MR 97e:58064

[36] D.Ruelle and D.Sullivan Currents, flows, and diffeomorphisms. Topology 14, 319-327, 1975. MR 54:3759

[37] I. Satake. On a generalization of the notion of manifold. Proc. Nat. Acad. Sci. U.S.A., 42:359363, 1956. MR 18:144a

[38] R. Schultz. Homotopy invariants and $G$-manifolds: a look at the past fifteen years. In Group actions on manifolds (Boulder, Colo., 1983), Contemp. Math Vol. 36, pages 17-81, 1985. Amer. Math. Soc., Providence, RI. MR 86e:57037

[39] D. Sullivan. A counterexample to the periodic orbit conjecture. Publ. Math. Inst. Hautes Etudes Sci., 46:5-14, 1976. MR 58:18492]

[40] W. P. Thurston. The Geometry and Topology of Three-Manifolds. Lecture notes, Princeton University, Princeton, N.J. 1979.

[41] T. tom Dieck. Transformation groups. Walter de Gruyter \& Co., Berlin, 1987. MR 89c:57048

[42] K. Varadarajan. On fibrations and category. Math. Zeitschrift, 88:267-273, 1965. MR 31:5199

[43] E. Vogt. Foliations of codimension 2 with all leaves compact. Manuscripta Math., 18:187-212, 1976. MR 53:4091

[44] E. Vogt. Foliations of codimension 2 on closed 3, 4 and 5-manifolds. Math. Zeit., 157:201-223, 1977. MR 57:17665

[45] E. Vogt. A periodic flow with infinite Epstein hierarchy. Manuscripta Math., 22:403-412, 1977. MR 57:17664

Department of Mathematics, University of Illinois at Chicago, 322 SeO (M/C 249), 851 S. Morgan Street, Chicago, Illinois 60607-7045

E-mail address: hcolman@math.uic.edu

URL: http://www.math.uic.edu/ ${ }^{\text {hcolman }}$

Department of Mathematics, University of Illinois at Chichgo, 322 SEO (M/C 249), 851 S. Morgan Street, Chicago, Illinois 60607-7045

E-mail address: hurder@uic.edu

$U R L:$ http://www.math.uic.edu/ ${ }^{\sim}$ hurder 